\title{
Artikel
}

Antje Sablotny*

\section{"Das mustu gleuben, oder der Teufel bescheisset dich." Die invektiven Paratexte der protestantischen Lügenden und ihre gattungskommunikative Funktion}


Abstract: The article deals with the Protestant genre 'Lügende' (word combined from 'Legende' [legend] and 'Lüge' [lie]) as a disparagement of Roman Catholic legends in the 16th century. The investigation concentrates on paratextualisation as elementary invective mode of 'Lügenden'. The analytical focus on titles, marginalia and so called 'reminders' ('Erinnerungen') shows the correlation between the generic term 'Legende' resp. 'Lügende' and the invective pattern of language use 'Lügende'. According to this, the article discusses 'Lügende' as a communicative genre. Furthermore, by understanding 'Lügende' as a meta genre, whose paratexts are its basic elements of metaization, paratexts refer to text transgressions. Therefore, they are specified as secondary forms of religious communication during the denominational conflicts and negotiation processes in the 16th century.

Keywords: Legende, Lügende, Luther, Paratext, Invektivität, Sprachgebrauchsmuster, kommunikative Gattung, Metagattung, religiöse Kommunikation - legendary tales, ,Lügende', Luther, paratext, invectivity, patterns of language use, communicative genre, meta-genre, religious communication

*Dr. Antje Sablotny, TU Dresden, SFB 1285, TP E, Antje.Sablotny@tu-dresden.de

\section{Hinführung}

Innerhalb der reformatorischen Auseinandersetzungen des 16. Jahrhunderts sind Herabsetzungen von Texten und Gattungen, die für die religiöse Kommunikation grundsätzlich relevant sind, zentral. Hier kommen sehr unterschiedliche Praktiken zum Tragen; es lässt sich jedoch beobachten, dass die Paratextualisierung etwa von theologischen Traktaten, legendarischen Erzählungen oder päpstlichen Bullen eine signifikante Rolle innerhalb dieser invektiven Praktiken spielt. Im Falle der Legenden, welche im Ausgang des Mittelalters eine Hochzeit erlebten, bewirken invektive Paratexte sehr spezifische gattungsdynamische und zugleich religiös-kommunikative Aushandlungsprozesse, welche sich in der protestantischen Metagattung der ,Lügende' verdichten. Mit Titel, Vor- und Nachwort, Marginalglossen und sogenannten Erinnerungen, die wiederum als etwas umfangreichere Kontextglossen aufgefasst werden können, versehen die Herausgeber der Lügenden altgläubige Mirakel- und Legendenerzählungen und setzen diese in ihrer Bedeutung und Funktion für die Heiligenverehrung und damit generell den Heiligenkult nachdrücklich herab.
Die Prägung des Begriffes, Paratext' sowie grundsätzliche Überlegungen zu Typologie und Funktionen des Paratextes gehen bekanntlich zurück auf den französischen Literaturwissenschaftler Gérard Genette: In seiner 1987 erschienenen Studie mit dem Titel Seuils hat er sich ausführlich mit den Beziehungen zwischen Text, Paratext, Autor:in und Leser:in beschäftigt - in einem Buch, das er selbst als „Buch vom Beiwerk des Buches" bezeichnet hat. ${ }^{1}$ Im Grunde gebe es kein Buch ohne verbale oder nonverbale Schwellen, die seine Rezeption steuerten.

Der Paratext ist [...] jenes Beiwerk, durch das ein Text zum Buch wird und als solches vor die Leser und, allgemeiner, vor die Öffentlichkeit tritt. Dabei handelt es sich weniger um eine Schranke oder eine undurchlässige Grenze als um eine Schwelle oder [...] um ein ,Vestibül', das jedem die Möglichkeit zum Eintreten oder Umkehren bietet; um eine ,unbestimmte Zone' zwischen innen und außen, die selbst wieder keine

1 Genette (2001) Paratexte. Paratextualität gehört neben Intertextualität, Metatextualität, Hypertextualität und Architextualität zu den fünf Formen textübergreifender Beziehungen im Rahmen von Genettes 1982 entwickelten Transtextualitätsmodells. Vgl. Genette (1993) Palimpseste. 
feste Grenze nach innen (zum Text) und nach außen (dem Diskurs der Welt über den Text) aufweist. ${ }^{2}$

Gleichsam sei diese Zone des Paratextes eine der ,Transaktion', weil dort der Ort bzw. der Raum für die bei Genette stets auf Autor:in oder Verleger:in zurückgehende Beeinflussung der Lektüre ist. Paratexte sind hier nicht nur medial greifbare Formen der Textpräsentation, sondern auch Diskurse und Praktiken der Rezeptionssteuerung. ${ }^{3}$ Insbesondere Genettes funktionsorientierte Fragen sind für die protestantischen Lügenden und ihre im Umfeld der wiederabgedruckten und kritisch kommentierten legendarischen Erzählung situierten Epitexte anschlussfähig. Hierzu gehört die illokutionäre oder perlokutionäre Wirkung von Paratexten, die je nach Intensität der Wirkung zwischen Information, Absicht, Interpretation, Entscheidung, Verpflichtung oder Anweisung unterschieden werden kann. ${ }^{4}$ Gerade in Bezug auf das im vorliegenden Beitrag fokussierte Verhältnis von Paratext und Gattung im Sinne paratextueller Einschreibung in Gattungs- und mithin Rezeptionshorizonte ist dieser Aspekt entscheidend. Mit dem funktionsorientierten Fragefokus erübrigen sich zugleich die immer wieder laut gewordenen Bedenken dagegen, die vornehmlich an Beispielen fiktionaler Erzählliteratur aus dem abendländischen Buchzeitalter entwickelte Systematisierung des Paratextphänomens auf mittelalterliche Handschriften und frühneuzeitliche Drucke anzuwenden. ${ }^{5}$ Als hermeneutisches Ver-

2 Genette (2001) Paratexte, S. 10.

3 Vgl. Genette (2001) Paratexte, S. 10.

4 Vgl. Genette (2001) Paratexte, S. 15-18, hier S. 18: „Manche Elemente besitzen sogar jene Macht, die die Logiker als performativ bezeichnen, das heißt die Macht zum Vollzug des Beschriebenen (,Ich eröffne die Sitzung'): Das gilt für Widmungen. Bei der Zueignung oder der Widmung eines Buches an Sowieso geschieht natürlich nichts anderes, als daß man auf eine der Seiten eine Wendung schreibt oder druckt wie: ,Für Sowieso'. Hier liegt ein Grenzfall der paratextuellen Wirksamkeit vor, da das bloße Sagen bereits das Tun ist. Das haftet jedoch auch bereits der Entscheidung für einen Titel oder der Wahl eines Pseudonyms an, den mimetischen Handlungen jeder schöpferischen Fähigkeit."

5 So hat schon Münkler (2000) Erfahrung, S. 244-266, in ihrer Arbeit zu den Orientreiseberichten des 13. und 14. Jahrhunderts die Übertragbarkeit von Genettes theoretischen Überlegungen zum Paratext auf mittelalterliche Handschriften thematisiert und dabei die Frage der Gattungskonstitution aufgeworfen. Sie konstatiert bspw. den elementaren Zusammenhang von Autornennungen bei der fahren stellt die Paratextanalyse freilich „kein one size fits all-Schema zur Verfügung, sondern einen Analyseansatz, der je nach Gegenstand und Frageinteresse in sehr unterschiedliche Kontexte auszugreifen vermag." ${ }^{6}$ Für die reformatorischen Lügenden werden ihre Paratexte zu den elementaren Trägern der Metagattung, mit denen nichts weniger als die (im Sinne der Protestanten vermeintliche) Wahrheit der Legenden, ausgetrieben' wird - es kann ihnen daher im Anschluss an Schlesier eine apotropäische Funktion zugewiesen werden. ${ }^{7}$ Diese aggressive Form paratextueller Neuperspektivierung eines Textes oder gar einer ganzen Gattung im reformatorischen Kontext ist kein Sonderfall. Es gibt einige Beispiele für invektiv paratextualisierte ,Editionen': Einen spektakuIären Auftakt bildet Ulrich von Hutten mit seiner Paratextualisierung der Bulle Exsurge Domine von 1520. Kurz nach dem Erscheinen ließ er den päpstlichen, um invektive Paratexte erweiterten Erlass in Straßburg nachdrucken, d.h. die Bulle erhält ein anders gestaltetes Titelblatt, ein Motto, zwei Widmungen, eine Vorrede sowie Glossierungen. ${ }^{8}$ Schon am Titelblatt wird das durch invektive Paratexte gestörte Verhältnis zwischen der Gattung, dem durch sie formulierten Geltungsanspruch sowie ihrer Pragmatik deutlich. Hutten hat hier u.a. das Wort "BVLLA" als generisches und autoritatives Signum vermittels Typographie und Layout im Vergleich zur Vorlage hervorgeho-

Beschreibung des Fremden nicht nur im Prolog, sondern schon im Incipit. Sie gehören zu den Gelingensbedingungen der Gattung des Augenzeugenberichts, der - so autorisiert - vom Fremden erzählen kann. Vgl. zur Anwendbarkeit von Genettes Konzept des Paratexts auf die frühneuzeitliche Literatur grundsätzlich auch der Band Pluralisierungen des Paratextes, hg. von Ammon/Vögel (2008), dessen Beiträge den gewinnbringenden Anschluss an Genette für den bei ihm ausgeklammerten Bereich vormoderner bzw. frühneuzeitlicher Literatur vor Augen führen. Dagegen plädiert bspw. Ott (2010) Erfindung, der sich kritisch mit den Prämissen des Sammelbands auseinandersetzt, für eine stärkere Reflexion und Historisierung des Paratextbegriffs für die Drucke der Frühen Neuzeit; er scheint dabei allerdings die bei Genette schon angelegte Flexibilität des Paratextkonzepts zu übersehen.

6 Stanitzek (2013) Paratextanalyse, S. 201. Zum Verdienst Genettes hinsichtlich der Paratextforschung vgl. auch Kuhn (2018) Wahre Geschichten, S. 20 und $25 f$.

7 Vgl. Schlesier (1993) Art. Apotropäisch; siehe auch die Anwendung bei Münkler (2019) Luthers Rom.

8 Eine ausführliche Analyse der paratextualisierten Bulle bietet Ammon (2006) „Bevor wir Dich hören, Heiligster." 
ben: Statt des Titels Bulla contra errores Martini Lutheri et sequacium in gleicher Schriftgröße wird nun "BVLLA" mit größerem Schriftgrad auf der ersten Zeile exponiert, in der zweiten und dritten Zeile folgt dann in Erweiterung der Vorlage "Decimi Leonis, contra errores Martini Lutheri, \& sequacium." Damit wird zunächst auf genau jenen Zusammenhang zwischen dem Machtanspruch des Papstes und dessen medialem Vollzug durch die Gattung ,Bulle' hingewiesen. Aus der Exponierung von ,Bulla' ergibt sich aber zugleich die herabsetzende Wirkung der paratextuellen Erweiterung und Transformation, deren Potential sich aus der Doppelbedeutung von ,Bulla' als päpstlicher Erlass einerseits und ,Wasserblase' andererseits speist. ${ }^{9}$ Die von Hutten hinzugefügte Anrede an den Leser, mit der eine neue Kommunikationssituation geschaffen wird, torpediert den eigentlichen päpstlichen Kommunikationskanal der Bulle. „Ihre Pragmatik wird somit empfindlich gestört und von einer neuen überlagert." 10 Auch Martin Luther hat schon lange vor seiner 1537 in Wittenberg gedruckten Lügend von St. Johanne Chrysostomo 1522 die immer wieder ergänzte päpstliche Bulla coenae domini von 1521 in derselben Absicht zunächst ins Deutsche übersetzt und dann in ähnlicher Weise paratextualisiert, wobei die Übersetzung freilich eigene deutungsverengende Akzente setzt - der Titel bei Luther heißt entsprechend Bulla Coenae domine, das ist, die Bulla vom Abendfressen des allerheyligsten Herrn, des Papstes. ${ }^{11}$ Dass die sogenannte Abendmahlsbulle oder Gründonnerstagsbulle Häretikern, Kritikern des Papstes sowie (Ver-)Fälschern päpstlicher Bullen die Exkommunikation androht, entbehrt dabei nicht einer gewissen Komik.

Im Falle der paratextuellen Zurichtung legendarischer Erzählungen jedoch erreicht das herabsetzende Verfahren eine besondere Tragweite, die sich nicht zuletzt in der Ausbildung einer neuen Gattung, der Metagattung Lügende niederschlägt. Natürlich ist die massive Infragestellung mithin die Destruktion päpstlicher Autorität

9 Vgl. Ammon (2006) „Bevor wir Dich hören, Heiligster.", S. 32.

10 Ammon (2006) "Bevor wir Dich hören, Heiligster.", S. $32 \mathrm{f}$.

11 Luther (1522) Bulla vom Abendfressen. Vgl. dazu Ammon (2007) „Quis dubitat de illo?", S. 285-291. Zur päpstlichen Generalexkommunikation vgl. Jaser (2016) Ostensio exclusionis. durch die paratextualisierten Bullen enorm, die Lügende aber setzt noch viel grundlegender an den Grundfesten religiöser Kommunikation und ihren Geltungsbedingungen an. Die Herabsetzung des legendarischen Erzählens zielt nämlich weiter als auf das Herabsetzen einer Erzählpraxis und ihrer Narrative. Sie stellt darüber hinaus die kirchliche Institution in Frage, insofern sie die Funktionalisierung des Heiligenkults im Rahmen von Ablass- und Wallfahrtswesen verurteilt und das kirchliche Recht am Gnadenschatz Christi entschieden zurückweist. Darüber hinaus diskreditiert die Lügende die Heiligenverehrung als eitle Werkgerechtigkeit, Fürbittgebete als Abgötterei und irritiert damit massiv die im Leben der Gläubigen stark verankerte Frömmigkeitspraxis. Dazu bedient sie sich fast ausschließlich invektiver Paratexte.

Dabei spielen Anmerkungen in den Marginalien eine besondere Rolle. Zwar sind ironische oder derb-provokative Textkommentierungen am Rande insbesondere in Form „visuelle[r] Tabubrüche" vor der Reformation bekannt. ${ }^{12}$ Der invektiv funktionalisierte Umgang Huttens oder Luthers mit marginalen (Para-)Texten unterscheidet sich aber erheblich von der antiken und mittelalterlichen Annotationspraxis. ${ }^{13}$ Grundsätzlich haben Marginalien in Handschriften die Funktion, „Begriffe und Inhalte des gegenüberliegenden Textes oder Bildes [zu] erläutern, übersetzen und erweitern sowie damit als ein mehr oder weniger geplantes Reservoir von präzisierendem und zusätzlich abrufbarem Wissen [zu] dienen." ${ }^{14}$ In ihrem Überblick über mittelalterliche und frühneuzeitliche Annotationspraktiken stellt Moulin nicht nur ab auf analytische Beschrei-

12 Vgl. Kato (2015) Versehren, S. 284-287, hier S. 285. Vgl. auch Klein (2007) Rand- oder Schwellenphänomen.

13 Vgl. Ammon (2007) "Quis dubitat de illo?", S. 285; Ammon/Vögel (2008) Pluralisierung des Paratextes, S. XIV. "Die wilde und ungeordnete Usurpation der Ränder in der gotischen Buchmalerei geschieht ganz bewusst, jedoch immer im Bewusstsein, dass der Werktext - ,the always already written Word' (Camille 1992: 22) - vorgegeben und in seiner Existenz, Lesart und Rezeption unumstößlich ist. Gerade deshalb können die Freiheiten, die sich der Illustrator mitunter nimmt, überhaupt so schamlos und unanständig sein, untergraben sie doch die Autorität des Werks, sei es geistlicher oder weltlicher Natur, in kein[]er Weise." Kato (2015) Versehren, S. 285.

14 Carmassi/Heitzmann (2019) Marginalien/Einleitung, S. 9. 
bungskriterien ${ }^{15}$ für Marginalien in Handschriften und Drucken, sondern auch auf funktionale Kontinuitäten - „wie etwa eine intensive Textentzifferung mit entsprechender sprachlich-inhaltlicher Erschließungsarbeit, die Auseinandersetzung mit fremd- oder fachsprachlichem Wortschatz, die kommentierende Begleitung des Gelesenen oder die Extraktion von Wissen". ${ }^{16}$ Annotationen - so Moulin präzisierend - tragen nicht nur zum Verständnis des kommentierten Textes bei. Mit ihnen wird der Basistext zudem ,erarbeitet', visualisiert, fortgeschrieben, werden „Haltungen (zum Gelesenen oder zur Lesesituation)" ausgedrückt, dabei "Netzwerke durch Texte hindurch und mit Texten [ge]schaffen" und schließlich wird das "Buchobjekt als Behälter" genutzt. ${ }^{17}$ Dem skizzierten Funktionspotential der Marginalien liegt ein - wie es Giuriato unter Rückgriff auf Gumbrecht formuliert - „stille[r] Imperativ" zugrunde, die leergelassenen Ränder und Zwischenräume zu füllen. ${ }^{18}$

Die Affordanz ${ }^{19}$ der Leere etabliert denn auch einen "Schauplatz subversiver Bemerkungen und Gedankenspiele", ${ }^{20}$ der im Zuge (experimenteller) bimedialer Phänomene der Schwellenzeit um 1500 und darüber hinaus an Kontur gewinnt und

15 Zu den ,Symptomwerten der Marginalität' vgl. Moulin (2019) Rand und Band, S. 26-29.

16 Moulin (2019) Rand und Band, S. 23.

17 Vgl. Moulin (2019) Rand und Band, S. 38-58, hier werden die Zwischenüberschriften zitiert.

18 Giuriato (2008) Prolegomena zur Marginalie, S. $178 f$. Vgl. auch Moulin (2019), S. 23. Zur ursprünglichen Bedeutung und zu eher pragmatischen Funktionen von leeren Seitenrändern und Zwischenräumen auf Schriftrollen und in Handschriften vgl. Kato (2015) Versehren, S. 282-284.

19 Der Begriff der, affordance' stammt ursprünglich aus der Wahrnehmungspsychologie. Gibson versteht darunter ein nicht unmittelbar sichtbares Handlungsangebot von Objekten in der Umwelt für das Individuum. Diese Wahrnehmung von Handlungsoptionen bestimme die Relation zwischen Umwelt und individuellen Beobachtenden, so dass Affordanzen von Objekten invariant sein können. Das Affordanz-Konzept wurde vom Designtheoretiker Donald A. Norman weiterentwickelt und in dieser Form breit rezipiert und fand darüber Eingang in die kultur-, insbesondere in die sozial- und medienwissenschaftlichen Arbeiten. Vgl. den Überblick zu Ursprung, Definition, Weiterentwicklung und Kritik des Affordanz-Begriffes bei Zillien (2019) Affordanz, S. 226f. Vgl. zudem die Beiträge von Dröse und Kanzler im vorliegenden Sonderheft.

20 Kato (2015) Versehren, S. 281. zu einem wesentlichen Ort konfessioneller Auseinandersetzungen wird. ${ }^{21}$

Der Paratext kann seinen Bezugstext relativieren, ironisieren oder gar destruieren, seine poetologische Faktur offenlegen oder über so zentrale Kategorien wie Fiktionalität, Autorität und Autorschaft Auskunft geben. [...] Gerade in der paratextuellen Zone, am vermeintlichen Rand des Textes, ereignet sich doch immer wieder Entscheidendes. ${ }^{22}$

Dieser Befund von Hamm gilt in besonderem Maße für die protestantische Gattung der Lügende. Hier zeigt sich der Paratext als eine Arena des Invektiven. ${ }^{23}$

\section{Thesen und zugrundeliegende Gattungskonzepte}

Meine Überlegungen zur ,Lügende' sollen nicht nur zeigen, dass Paratexte in den konfessionellen Auseinandersetzungen des 16 . Jahrhunderts entscheidende kommunikative Formen des Invektiven darstellen. Mit der Paratextualisierung liegt vielmehr ein wirkmächtiges invektives Verfahren vor, weil mit ihr die Grenzen legendarischen Erzählens überschritten werden: Einerseits evozieren die Paratexte Gattungsreflexionen und haben Teil am Gattungsdiskurs der Legende. Andererseits etablieren sie einen spezifischen invektiven Umgang mit den durch das legendarische Erzählen tradierten Glaubensprämissen auch außerhalb ihrer genuinen Kommunikationssituation. Invektive Anschlusskommunikation wird so dynamisiert. Aus systemtheoretisch geprägter kommunikationstheoretischer Sicht: Die Lügenden übernehmen eine signifikante Funktion in der Re-Organisation des religiösen Systems während seiner Ausdifferenzierung im 16. Jahrhundert.

Nach einem knappen literaturgeschichtlichen Überblick zur ,Lügende' (3.), der die Grundzüge der protestantischen Metagattung und das Textkorpus vorstellt, sollen zunächst thematische und rhematische Implikationen des Titels am Bei-

$21 \mathrm{Zu}$ mediengeschichtlichen Aspekten frühneuzeitlicher Paratextualität am Bsp. von Sebastian Brants Vergilius pictus (1502) vgl. Hamm (2017) Zu Paratextualität.

22 Hamm (2017) Zu Paratextualität, S. $238 f$.

$23 \mathrm{Vgl}$. Ellerbrock/Koch/Müller-Mall et al. (2017) Invektivität, S. 12-15. 
spiel der lutherischen Lügend von St. Johanne Chrysostomo erörtert werden (4.), um einerseits das Wechselverhältnis zwischen der Gattungsbezeichnung und dem invektiven Sprachgebrauchsmuster ,Lügende' zu verdeutlichen und andererseits die Wirkung der Lügende als kommunikative Gattung zu erläutern. Mit der Präzisierung der Lügende als einer Metagattung, deren zentrales Metaisierungsverfahren sich in den Paratexten zeigt, soll deutlich werden, dass Titel, Randglossen und Erinnerungen weniger ,Beiwerk', als vielmehr gattungskonstitutive Elemente darstellen (5.). Als solche verweisen sie nicht auf ein Problem, sondern auf das Potential der durchlässigen Grenze zwischen Text und Paratext für die Analyse. Schließlich werde ich die Paratexte in ihrer Bestimmung als sekundäre Formen religiöser Kommunikation in den konfessionellen Konflikten und Aushandlungsprozessen des 16. Jahrhunderts konkretisieren (6.).

In der Analyse nicht berücksichtigt werden die entweder wenig umfänglichen oder erst gar nicht enthaltenen Widmungen in den Lügenden, weil sie für die Gattungsfrage nicht relevant sind. In den Vor- bzw. Nachreden lassen sich dagegen durchaus auch rezeptionslenkende Gattungsreflexionen beobachten: Das lässt sich insbesondere für Luthers Nachrede zur Lügend von St. Johanne Chrysostomo zeigen, in der er seine Lügentypologie entfaltet: $\mathrm{Er}$ unterscheidet die das Seelenheil kostenden teuflischen Lügen von den harmlosen Lügen, zu denen Luther wiederum leicht durchschaubare Lügen zum Zwecke der Unterhaltung zählt. Hier liegt denn auch das Deutungspotential der Metagattung, die altgläubige Legende im Sinne ihrer Fiktionalisierung zu ,entschärfen' (und herabzusetzen) und in einen neuen Gebrauchskontext - der unterhaltenden Literatur - zu transferieren. ${ }^{24}$

In den nachlutherischen Lügenden-Vorreden indes werden über die Bezeichnung der Legenden als Lügenden hinaus, wie sie bereits die Titel vornehmen, keine weiteren signifikanten gattungsreflexiven Gedanken entwickelt. Sie werden daher keinen Analyseschwerpunkt bilden. Verwiesen sei hierfür zunächst auf die Vorrede zur ersten Lügenden-Ausgabe Hieronymus Rau-

24 Dazu Sablotny (2019) Metalegende, S. 180-183; ferner Münkler (2015) Legende/Lügende sowie Ziegeler (1999) Wahrheiten, S. 243-245. schers, ${ }^{25}$ in der der erst lutherische, dann aber zum katholischen Glauben konvertierte Theologe Friedrich Staphylus als Judas inszeniert und u.a. als "abtrünniger Mameluck" herabgesetzt sowie Luther gegen dessen Kritik verteidigt wird. Erst gegen Ende der Vorrede heißt es, Staphylus habe ihn "gleich lustig gemachet", die folgenden Lügen dezidiert zur Unterhaltung der katholischen Gegner zusammenzustellen, was freilich ironisch gemeint ist. Der Begriff ,Lügende' wird dabei allerdings nicht verwendet. Das gilt auch für die Vorrede zur zweiten Centurie, ${ }^{26}$ die im Rahmen der (Gegen-)Invektiven gegen Staphylus, Bruder Nardian aus Regensburg (Johann Albrecht) und den Theologieprofessor Martin Eisengrein, den "Lugenhafftigen Miracklen" und "offentliche[n] Lugen", die herabsetzenden Begriffe für Rauschers Lügenden von Seiten der Altgläubigen, wie "Centonouela", „Erdicht Fablen", „Gedicht" oder "Zotten", gleichsam zur Seite stellen und somit den Raum für Gattungskonnotationen öffnen. Rauschers Auseinandersetzung mit den Diffamierungen "Bruder Rausch" und „Fabel hanssen", auf die er in dieser Vorrede zur zweiten Centurie reagiert, zielen in dieselbe Richtung. ${ }^{27}$ In den Vorreden Rauschers dominiert also der persönliche Schlagabtausch zwischen den konfessionellen Gegnern, bei dem die Überschreibung der Legende als Lügende immer schon vorausgesetzt und kontinuierlich wiederholt wird. Der Begriff ,Lügende' wird dabei gar nicht oder kaum gebraucht - bspw. aber in der Vorrede zur vierten Centurie, in der von "Lugenhafftiger Lugend" die Rede ist. ${ }^{28}$ Hinsichtlich der Gattungsthematik bieten die Vorreden jedoch keine neuen Aspekte. ${ }^{29}$

Um die Funktionen invektiver Paratexte in der protestantischen Lügende spezifizieren zu können, werden verschiedene gleichwohl miteinander verschränkte Gattungskonzepte mit kommunika-

25 Rauscher (1562) Papistische Lügen I.

26 Rauscher (1564) Papistische Lügen II.

27 Bruder Rausch ist eine um 1488 erstmals gedruckte Teufelserzählung, in der der Teufel unter dem Namen Bruder Rausch in einem Kloster sein Unwesen treibt.

28 Rauscher (1564) Papistische Lügen IV.

29 Sie sind aber bedeutsam für die kommunikative Praxis der Grenzziehung in den Lügenden. Vgl. Sablotny (im Erscheinen) Doing the Other. Zur Funktion der Vorrede im 16. Jahrhundert vgl. immer noch grundlegend Schwitzgebel (1996) Vorrede, die sich u.a. mit den protestantischen Exempelsammlungen Caspar Goltwurms und Andreas Hondorffs auseinandersetzt (ebd., S. 11-56). 
tionstheoretischen Überlegungen Luhmann'scher Prägung verknüpft. Gemeint ist zunächst der ,klassische' textsortensystematische Gattungsbegriff, der literarische Texte nach inhaltlichen und formalen Kriterien unterscheidet. Die Rekonstruktion von Reihenbildungen orientieren sich bereits seit längerem nicht mehr an der von Aristoteles und Goethe geprägten Dreiteilung von Epik, Lyrik und Dramatik, die freilich im Hintergrund immer noch eine Rolle spielt - wie etwa bei Hempfer, der historisch konkrete Gattungen einerseits und transhistorische Schreibweisen andererseits unterscheidet und das Epische, Lyrische und Dramatische als primäre Schreibweisen versteht. ${ }^{30}$ Mit Voßkamp lassen sich Gattungen als „institutionalisierte Organisationsformen literarischer Kommunikation" und "soziokulturelle Phänomene" verstehen. ${ }^{31}$ Hier schließen sich unmittelbar Fragen nach dem Funktionspotential von Gattungen an. Der Angriff der Lügende auf legendarische Erzählmuster irritiert religionspraktische und institutionelle Funktionen religiöser Gebrauchsliteratur; die Lügende ist daher generischer Ausdruck des durch die Reformation veränderten Umgangs mit den Heiligen. Der auf Iterativität angelegte Gebrauch des Wortes ,Lügende' verdeutlicht diesen Zusammenhang. Es bildet sich so eine auf inhaltliche, formale und funktionale Bündelungen bezogene Reihe, deren wesentliches Merkmal die invektive Paratextualisierung legendarischer Erzählungen darstellt. Damit vollzieht sich ein Prozess der Gattungsbildung, die hier mit der Gattungstransformation von der Legende zur ,Lügende' einhergeht. In diesem Sinne lässt sich an den im vorliegendem Sonderheft von Meier-Vieracker vorgestellten rezeptions- und diskursorientierten Gattungsansatz des Doing Genre anschließen: Die Legende erhält über die Bezeichnung ,Lügende' eine metapragmatische Rahmung und wird so zu einer anderen Gattung transformiert. Wie schon am ,Gattungsbegriff selbst abzulesen, ist allerdings die genuine und konstitutive Bezugnahme auf bereits etablierte Muster des legendarischen Erzählens für die Lügende zentral. Diese elemen-

30 Zum literaturwissenschaftlichen Gattungskonzept vgl. grundsätzlich Zymner (2003) Gattungstheorie; Hempfer (1973) Gattungstheorie.

31 Voßkamp (1990) Utopie, S. 265, sowie Voßkamp (1977) Gattungen, S. 27. Siehe hierzu auch den einleitenden Beitrag von Marina Münkler. tare Bezogenheit auf eine vorgängige Gattung, mit der sich die Lügende wesentlich von dem von Meier-Vieracker vorgeführte Doing der Wutrede unterscheidet, ist prägnanter im Konzept der Metagattung zu fassen, ${ }^{32}$ das maßgeblich auf Wolf zurückgeht. ${ }^{33}$ Bei einer Metagattung handelt es sich um eine Gattung über eine Gattung. Über unterschiedliche Metaisierungsverfahren, etwa im Rahmen von Kommentaren oder Beschreibungen, sollen Reflexionen über die ,Gemachtheit' der mit ihr fokussierten Gattung ausgelöst werden, welche wiederum längerfristige gattungsinnovative bzw. -dynamische Prozesse initiieren können. Im Gegensatz zu modernen Metagattungen, die mit Illusionsstörungen operieren, soll mit den invektiven Paratexten der Lügende die Wahrhaftigkeit des legendarischen Erzählens nachhaltig zerstört werden. ${ }^{34}$ Damit nimmt die Lügende erheblichen Einfluss auf die für die religiöse Gattung der Legende höchstrelevante pragmatische Dimension. In diesem Sinne überschreiten ihre invektiven Paratexte die textimmanenten Gattungsgrenzen nicht nur hin zum Diskurs über Formen und Funktionen altgläubiger Heiligenverehrung, sondern haben auch das Potential, die mit dem legendarischen Erzählen verknüpfte Frömmigkeitspraxis zu stören. Den letzten pragmatischen Aspekt möchte ich schließlich anhand des soziolinguistisch geprägten Modells der kommunikativen Gattungen reflektieren, die als musterhaft verfestigte Formen alltagssprachlicher Kommunikation aufgefasst werden. Mittels der Konzeption der kommunikativen Gattung kann einerseits das Wechselspiel von Sprachgebrauchsmuster und Textsorte ,Lügende' gezeigt werden, andererseits kann es das Abarbeiten am kommunikativen Problem auf Seiten der Protestanten verdeutlichen: die Herabsetzung des Heiligenkults ohne Diskreditierung der Heiligen. Etabliert werden soll die funktionelle Herabsetzung der Heiligen von vermeintlichen ,Abgöttern' hin zu vorbildlichen und tröstenden Exempeln. Von Ausnahmen abgesehen, soll dabei nicht ihre Existenz und Heiligkeit in Frage gestellt werden.

32 Keinesfalls ist die Lügende bloß als "eine Legende mit ,poetologischen' Aspekten" zu betrachten - so Ecker (1993) Legende, S. 243, in Anm. 902.

33 Vgl. Wolf (1993) Ästhetische Illusion, sowie Wolf (2007) Metaisierung.

34 Vgl. Sablotny (2019) Metalegende. 


\section{Die protestantische ,Lügende'}

Bei der Lügende handelt es sich um eine relativ kurzlebige protestantische Metagattung von der ersten Hälfte des 16. bis zum beginnenden 17. Jahrhunderts, die aus der Herabsetzung des altgläubigen Heiligenkults hervorgeht. Diese äußert sich in der immer radikaler werdenden Ablehnung gegenüber der Auffassung der Werkgerechtigkeit, gegenüber Ablasshandel, Wallfahrten, Heiligendienst und insbesondere gegenüber der Funktion der Heiligen, zwischen Gott und den Menschen zur Erlangung des Seelenheils zu vermitteln. ${ }^{35}$ Im Rahmen dieser Frömmigkeitspraktiken hat die Legende eine wichtige Funktion: Durch das Erzählen vom Heiligen produziert sie erst die Anschlussmöglichkeiten für religiöse Praktiken, die nicht ausschließlich durch das Heiligsprechungsverfahren instituiert und legitimiert werden können. In diesen Verfahren spielen legendarische Viten bereits eine zentrale Rolle. ${ }^{36}$ Die Lügende kann insofern nicht nur als Herabsetzung der katholischen Legende und ihrer Grundsätze, sondern auch der gesamten Frömmigkeitspraxis der Heiligenehrung dienen. ${ }^{37}$ In Bezug auf die Legenden nutzen Luther und seine Nachfolger nicht die sonst häufig eingesetzten Mittel der Parodie oder Travestie, um die vorliegenden Legenden und Legendensammlungen herabzusetzen, sondern die invektive Kommentierung: Die Legenden werden in der überlieferten Textgestalt abgedruckt, dann aber invektiv kommentiert - mit Vorwort, Nachwort, Marginalglossen und sogenannten Erinnerungen. Das prototypische Gattungsexemplar hat Martin Luther mit seiner Lügend von St. Johanne Chrysostomo 1537 zu Wittenberg in den Druck gegeben. ${ }^{38} \mathrm{Er}$ hat die Legende des Heiligen Johannes Chrysostomus

35 Vgl. Kaufmann (2015) Reformation der Heiligen, S. 216-223; Münkler (2015) Legende/Lügende, S. 125-129; Münkler (2008) Sündhaftigkeit, S. 40-46.

$36 \mathrm{Vgl}$. zu diesem Zusammenhang am Beispiel der Franziskuslegenden Münkler (2013) Amicus dei.

37 Zur Lügende vgl. Sablotny (im Erscheinen) Doing the Other, Sablotny (2021) corpus corruptum; Holtzhauer (2020) Destruktion; Sablotny (2019) Metalegende; Münkler (2015) Legende/Lügende; Ziegeler (1999) Wahrheiten; Schnyder (1979) Legendenpolemik; Schenda (1974) Hieronymus Rauscher; Schenda (1970) Legendenpolemik.

38 Vgl. Luther (1537) Die Lügend. Zum generischen Prototypenkonzept vgl. den Beitrag von Meier-Vieracker in diesem Sonderheft. aus dem Legendar Der Heiligen Leben entnommen, ${ }^{39}$ das um 1400 entstanden ist und zumindest bis weit ins Spätmittelalter sehr populär war. Die Legende des Heiligen Johannes Chrysostomus gehört zum Typus der Sünderheiligenlegende und zeichnet sich durch eine finale Erzählstruktur aus, die nach dem Muster von ,Erwählung - Sünde Buße - Erwählung' gebaut ist. ${ }^{40}$ Innerhalb dieses Erzählschemas bildet der Aspekt der Sünde auf der Ebene der histoire ein mit zahlreichen, als kontingent markierbaren Handlungen ausgestattetes und deshalb leicht irritierbares Element. So wird beispielsweise davon erzählt, dass die jungfräuliche Tochter des Kaisers während eines Spaziergangs im Wald plötzlich von einem starken Wind gepackt und zur Einsiedlerhöhle des Heiligen getragen wird - woraus dann die Verführung des keuschen Einsiedlers durch die nicht minder keusche Kaiserstochter resultiert. Mit der Randglosse "Je, das mus ja war sein, wer kundts erdencken!"41 markiert Luther das Erzählereignis als unwahrscheinlich und unglaubwürdig, zugleich macht er damit die altgläubigen Autoren solcher ,Lügenlegenden', ${ }^{42}$ aber auch die dieser Lektüre folgenden Rezipient:innen lächerlich.

Während von Luther selbst keine weiteren Lügenden überliefert sind, haben seine Anhänger eine Gattungstradition ausgeprägt: Der Lutherschüler Erasmus Alber(us) veröffentlichte 1542 seinen Barfuser Münche Eulenspiegel vnd Alcoran mit einer Vorrede Luthers; Albers Eulenspiegel vnd Alcoran bezieht sich maßgeblich auf das umfangreiche zwischen 1385 und 1390 entstandene Werk De conformitate vitae beati Francisci ad vitam Domini Iesu des Franziskanerbruders Bartholomaeus de Rinonico, markiert aber nur implizit die Gattung Lügende im Titel; die Gattungsmarkierung jedoch wird in den Vorworten nachgeholt. Während ,Eulenspiegel' auf die spätmittelalterliche und frühneuzeitliche Schwankliteratur rekurriert, konnotiert der (,türkische') ,Alcoran' Aberglauben und Götzenanbetung. Aus der titelgebenden Kombination beider Begriffe wird - so Alber in seinem Vorwort „an den Christ-

39 Brand/Jung/Williams-Krapp (2004) Der Heiligen Leben, hier Nr. 82, S. 434-441.

40 Vgl. Münkler (2015) Legende/Lügende, S. 134f.

41 Luther (1537) Die Lügend, S. 57.

42 Vgl. Münkler (2015) Legende/Lügende, S. 132. Der Transformationsprozess von Legende - Lügenlegende Lügende wird im vierten Abschnitt näher erläutert. 
lichen Leser" - das bei der Lektüre der altgläubigen Franziskus-Legenden ausgelöste Entsetzen, zugleich der sich einstellende Eindruck der Lächerlichkeit zum Ausdruck gebracht. Neben Pietro Paolo Vergerio ${ }^{43}$ ist die monumentale Ausgabe von fünf Bänden zu je etwa 100 Papistischen Lügen, die zwischen 1562 und 1564 durch Hieronymus Rauscher in den Druck gegeben wurden, nicht zuletzt ob ihres publizistischen Erfolgs hervorzuheben. ${ }^{44}$ Der vollständige Titel - Hundert außerwelte / grosse / vnuerschempte / feiste / wolgemeste / erstunckene / Papistische Lügen Welche aller Narren Lugend / als des Eulenspiegels [...] weit vbertreffen / damit die Papisten die fürnemsten Artickel ihrer Lehre verteidigen / die armen Christen aber verblenden / vnd in abgrund der Hellen verfüren [...] - zeigt einerseits das Changieren und mithin den synonymen Gebrauch von Lüge und Lügende, andererseits den hier stärker gesetzten Akzent auf den Vorwurf und die Enttarnung der ,Lügen' sowie auf die als bewusste Täuschungen markierten Erzählungen durch die Vertreter der Kirche in den konfessionellen Auseinandersetzungen um die religiöse Wahrheit. Das zeigt sich auch an der Durchnummerierung der Legenden- und Mirakelerzählungen bis zur „100. Bäpstisch Lug" in der ersten Centurie.

Die Lügenden werden von zahlreichen paratextuellen Elementen begleitet: Neben Vorworten, Nachworten und Marginalglossen gibt es sogenannte Erinnerungen als invektive Paratextelemente, die mit einer Zwischenüberschrift hervorgehoben werden und die jede einzelne Erzählung beschließen. Die Erinnerungen haben vorrangig die Funktion, die erzählten Ereignisse als unglaubwürdig herabzusetzen und diejenigen moralisch zu verurteilen, die solche Legendenerzählungen verbreiten und funktionalisieren. Oft werden Bibelzitate oder auch Redensarten und Sprichwörter hierfür autoritativ angeführt, von einer argumentativen Vorgehensweise kann gleichwohl nicht die Rede sein. Mit Blick auf die Unwahrheitsbehauptungen, Verurteilungen, Abwertungen, Vorwürfen, Beleidigungen und Verwünschungen bestimmt das Feld verdiktiver und konduktiver Äußerungen das Invektiv-Register der Erinnerungen. Man kann die Redundanz,

43 Vgl. Vergerio (1556) De Gregorio Papa.

44 Vgl. dazu, gleichwohl abschätzig, Schenda (1974) Hieronymus Rauscher, S. 255-258.
Undifferenziertheit oder argumentative Oberflächlichkeit in Rauschers Erinnerungen bemängeln. ${ }^{45}$ Das würde aber ihre relevante Funktion überdecken, ein in Relation zu den präsentierten Mirakelerzählungen textuelles Gegengewicht zu schaffen; im Gegensatz zu Albers Kontextglossen wird dieses Verfahren bis zum Schluss durchgeführt. Moralische Kommentare gehören zur homiletischen Praxis und finden sich - auch mit der Bezeichnung ,Erinnerung' - vorwiegend in Exempelsammlungen. ${ }^{46}$ Die Umsetzung paratextueller Erinnerungen liegt insofern nahe, als Rauscher vornehmlich auf spätmittelalterliche Predigtexempel zurückgreift. Die noch nicht durch eigene Überschriften abgesetzten Kontextglossen in Albers Alcoran, dessen Erzählungen Rauscher für das letzte Viertel seiner ersten Centurie wörtlich übernommen hat, ${ }^{47}$ mögen ihn zu den Erinnerungen angeregt haben. Ihre homiletische Funktion aber erfährt schon bei Alber unzweifelhaft eine Negation, weil den Mirakel-Exempeln als Lügen ihre Authentizität und mithin Vorbildhaftigkeit radikal abgesprochen wird. ${ }^{48}$

Dieser Befund trifft schließlich auch für die weniger derb-plakativen Erinnerungen Caspar Fincks zu. Der Gießener Theologieprofessor hat Rauschers Papistische Lügen 1614 und 1618 nicht zuletzt als Reaktion auf die Gegenreformation neu aufgelegt und im Zuge seiner Überarbeitung erweitert. ${ }^{49}$ In seinem Vorwort zur ersten Ausgabe von 1614 formuliert er die Gründe für die Neuauflage ein halbes Jahrhundert nach der letzten Ausgabe der Rauscher-Centurien. Sie sei notwendig,

45 Vgl. Schenda (1974) Hieronymus Rauscher, S. 252-255. 46 Im Kontext der Faustbuch-Transformationen durch Georg Rudolff Widman (1599) und Christian Nikolaus Pfitzer (1647), vgl. dazu Münkler (2011) Narrative Ambiguität, S. $178-181$ und S. $184 f$.

47 Zu den Quellen Rauschers vgl. Schenda (1974) Hieronymus Rauscher, S. 200-206.

$48 \mathrm{Vgl}$. ferner Holtzhauer (2020) Destruktion, Anm. 40 auf S. $103 f$.

$49 \mathrm{Vgl}$. Finck (1614/18) Papistische Lügen. „Im selben Jahr erschien übrigens auch eine Neuauflage von Albers Alcoran, ohne Ortsangabe. Sie enthält neben 595 Konformitäten-Exempeln ,Der Barfüser Münch. Zehen Gebot', einen Konformitäten-Vergleich zwischen S. Benedict und Elias sowie Fischarts ,Barfüsser Secten und Kuttenstreit'." Schenda (1974) Hieronymus Rauscher, S. 198, Anm. 94. 
weil noch heutiges Tages die Papisten solche Lügen vor wahr halten / vnnd ihnen / Gott erbarms / mehr glauben / als der heiligen Schrifft. Darnach weil das Bapstthumb hin vnd wider einreisset / vnd vberhandt nehmen wil. Zum dritten / weil sie sich wol kitzeln mit den Tischreden deß Herrn Lutheri seliger / vnd dieselbige wol vor Narrentheidinge außruffen / da doch ire Legenda anders nicht als rechte Thorenbossen seyn. Zum vierdten auch / weil sie sich vber die Protestirende beschweren / daß sie in den Legenden vnerfahren seyen $[\ldots] .{ }^{50}$

Vor allem mit dem ersten Aspekt, die ,Papisten' glaubten den Legenden mehr als der Heiligen Schrift, wird das für die Metagattung Lügende wesentliche Legitimationskontinuum aufgegriffen. Es ist fundiert in den theologischen sola-Grundsätzen Luthers, insbesondere in den Exklusivaussagen sola scriptura und solus Christus. Mit Blick auf die Divergenzen oder gar Irrtümer produzierende Praxis der Schriftauslegung fordert Luther die Exklusivität biblischer Autorität, ${ }^{51}$ was auch impliziert, von der bis dahin stark favorisierten Lektüre von Heiligenlegenden Abstand zu nehmen. Denn Legenden würden verehrungswürdige Heilige als Interzessoren zwischen Transzendenz und Immanenz inszenieren und die begehrte Fürsprache bei Gott stelle die göttliche Suprematie in Frage. Die Betonung dieses Aspekts bei Finck weist daher indirekt auf ein weiteres Kontinuum der Metagattung hin: auf das signifikante Problem der fest verankerten religiösen Praxis der Heiligenverehrung und -anrufung, das die Lügende zu bearbeiten versucht.

\section{Paratext und Gattung: Der Titel}

Paratextelemente stehen grundlegend in einem engen Zusammenhang mit Gattungen, weil sie oft die Einschreibung des durch sie spezifizierten Textes in eine Gattung überhaupt erst herstellen, selbst wenn sie diese nur vortäuschen oder durch

50 Finck (1614) Legendorum Papisticorum, Bl. iiiv und iiiir, VD17 1:076768U. Anders als in den folgenden Ausgaben, die Rauschers Titel aufgreifen, ist auf dem Titelblatt hier von "verlegene[n] Papistische[n] Unwahrheiten" die Rede. 51 Schon 1519, in der Bekräftigung aller Artikel Martin Luthers, die durch die Bulle Leo $X$. neuerlich verdammt worden sind (vgl. Luther, WA 7, S. 94-151) hat Luther seinen schrifthermeneutischen Grundsatz von der sich selbst auslegenden Schrift ausführlich erläutert. ihre kommunikativen Praktiken eine Gattung erst konstituieren:52 Gattungsangaben und Gattungskonnotationen (in umfangreicheren Paratexten auch Gattungsdefinitionen und -reflexionen) finden sich oft im Titel bzw. Untertitel. Er kann zu einem wichtigen Diskursort von Gattungen werden. ${ }^{53}$ Im Folgenden möchte ich diesen Zusammenhang zunächst anhand des Titels auf dem Titelblatt von Luthers Lügend veranschaulichen. ${ }^{54}$

Der zweite Teil des Titels, mit dem Luther seine Lügend von St. Johanne Chrysostomo bezeichnet, lässt sich insofern als eine thematische Information begreifen, als er Inhaltliches ankündigt: ${ }^{55} \mathrm{Es}$ geht um die Geschichte eines Heiligen mit Namen Johannes Chrysostomus, der zur Publikationszeit der Lügende beim Publikum als bekannt vorausgesetzt werden darf. Der Begriff der Lügende hingegen macht den Titel paratextuell gesehen komplex, weil hier mehrere funktionale Aspekte zusammenkommen und thematische mit rhema-

52 Vgl. zum letzten Aspekt der Gattungskonstituierung durch metapragmatische Rahmungen den Beitrag von Simon Meier-Vieracker im vorliegenden Sonderheft.

53 "Der Gattungsvertrag entsteht, mehr oder minder kohärent, durch die Gesamtheit des Paratextes und, umfassender, durch die Beziehung zwischen Text und Paratext." Genette (2001) Paratexte, S. 45. Kritisch zur Metapher des Fiktionsvertrags Kuhn (2018) Wahre Geschichten, S. 41-43. 54 "Das Titelblatt taucht erst in den Jahren 1475 bis 1480 auf und bleibt lange Zeit, bis zur Erfindung des gedruckten Umschlags, der einzige Anbringungsort für einen Titel, der [...] oft mit verschiedenen, für uns beiläufigen Angaben überladen ist." Genette (2001) Paratexte, S. 67. „Während Kolophone und Vorreden bereits in vielen spätmittelalterlichen Handschriften auftreten und sich die Drucker oder Herausgeber, die ja vielfach früher als Schreiber tätig waren, an diesen Traditionen orientieren konnten, entstand das Titelblatt erst mit dem gedruckten Buch; seine Herausbildung erfolgte daher langsamer." Wagner (2008) An der Wiege, S. 133. Zur Entwicklung und zu den Funktionen des Titelblatts vgl. unter Verweis auf die Studien von Smith (2000) und Rautenberg (2004) ebd., S. 140-148, hier S. 146: „Festzuhalten ist, daß Titelseiten als Paratexte vielfältige Funktionen erfüllen konnten: Schon in ihrer rudimentärsten Form, als leere Seite, schützen sie das Buch im Distributionsprozeß. Als, label-title' informieren sie den Drucker und Buchhändler über den Inhalt des Buchs und fungieren damit vor allem als Hilfsmittel bei der Verwaltung gedruckter Bücher. Angereichert um Zusatzinformationen, dienen sie als Einladung zur Lektüre und damit als Anreiz zum Kauf, also als ,Marketing'-Instrument." Zu Titel-Charakteristika vgl. auch Sánchez (1999) Titel, S. 249-253. 55 Zur Differenzierung von thematischen und rhematischen Titelangaben und deren Funktionen vgl. Genette (2001) Paratexte, S. 77-102. 
tischen Angaben kombiniert werden. In Bezug auf ihren deskriptiven Wert kann ,Lügende' zunächst auf thematische Angaben hinweisen, weil sie in unzweifelhaft pejorativer Absicht den folgenden Inhalt als Lüge und arglistige Täuschung wertet der Begriff konnotiert diese Aussage.

Invektive Namensverdrehungen und Wortspiele wie jene Verballhornung der Legende zur Lügende sind in den konfessionellen Auseinandersetzungen des 16 . Jahrhunderts populäre und wirkmächtige Verfahren zur Herabsetzung des jeweiligen konfessionellen Gegners: ,Luther' wird zu ,Lotter' oder (wieder) zu ,Luder', die päpstlichen ,Decretalen' zu ,Drecktalen', die Augsburger ,Konfession' zur ,Konfusion'. ${ }^{56}$ Mit der Lügende wird ein unmittelbarer Zusammenhang gestiftet zwischen dem religiös bestimmten Begriff der Lüge und der für die römisch-katholische Frömmigkeitspraxis wichtigen Gattung der Heiligenlegende - mit dem eindeutigen Zweck, altgläubige Legenden- und Mirakelerzählungen und insbesondere ihre Funktionalisierung durch die Kirche als lügenhaft bzw. verlogen zu diskreditieren.

Zwar beruht der christliche Glauben grundsätzlich auf dem Gegensatz von Gut und Böse sowie der göttlichen Wahrheit bzw. der Wahrheit des Glaubens und der Erkenntnis gegen teuflische Lüge, Täuschung und Verführung zur Sünde. ${ }^{57}$ Im Zeitalter der reformatorischen Auseinandersetzungen und der Konfessionalisierung aber gewann die Gegenüberstellung von Wahrheit und Lüge verstärkt argumentativ an Bedeutung und damit an Kontur. Ausdruck hierfür ist die zu beobachtende Tendenz konfessioneller Vereindeutigung und die Konjunktur des Lüge- und Täuschungsdiskurses, in dem nicht zuletzt Prozesse von Disambiguierung und Ambiguierung komplex zusammenlaufen. ${ }^{58}$ Die Diffamierung des konfes-

56 Vgl. Schenda (1974) Hieronymus Rauscher, S. 188 mit Anm. 19. Vgl. hierzu insbesondere den Beitrag von Jan Martin Lies in vorliegendem Band.

57 Zur ,Wahrheit' und ihrer Bedeutung im Alten und Neuen Testaments vgl. den Überblick bei Herms (2003) Wahrheit/Wahrhaftigkeit, S. 337-345; vgl. zudem Dietzsch (2001) Lüge, S. 20f.; Behringer (2005-12) Lüge, Abschnitt 2. Religiöse Ablehnung; Großhans/Sparn (2005-12) Wahrheit, Abschnitt 3. Religion und Theologie.

58 Vgl. Stollberg-Rilinger (2013) Einleitung, S. 9-15; Behringer (2005-12) Lüge, Abschnitte 3. Legitime Notwehr und 4. Verstellung als Gesellschaftsspiel; vgl. zudem Dietzsch (2001) Lüge, S. 21-24. sionellen Gegners als Lügner und seines Handelns als böswillige Täuschung erweist sich als beliebte, da wirkmächtige invektive Strategie, die der dialektischen Logik der Herabsetzung des Anderen und der Eigenprofilierung folgt und in der antiken Rhetorik ihre Wurzeln hat. ${ }^{59}$,Lügende' markiert daher nicht nur den bezeichneten Inhalt als besonders fragwürdig und scheinheilig, sondern immer zugleich auch dessen Urheber als Lügner, der in teuflischer Absicht zu täuschen versucht. ${ }^{60}$

Die titelgebende ,Lügende' ist darüber hinaus im rhematischen Sinne auf die Gattung bezogen: Einerseits wird mit ihr hypertextuell auf die religiöse Gattung der Legende zurückgegriffen; ${ }^{61}$ andererseits impliziert die Wortneuschöpfung ein gattungsinnovatives Potential. Durch die Produktion einer ganzen Reihe von Texten, die das Wortspiel aufnehmen, entwickelt sich die Lügende zu einer neuen Gattung. Hier sind die Centurien Rauschers nicht nur wegen ihrer Quantität hervorzuheben, sondern wegen ihrer generischen Engführung der ,Lügenlegenden' als Unterhaltungsliteratur. Die im Titel explizit realisierte Verknüpfung mit Schwankliteratur und Prosaromanen etabliert ein weiteres Element der Diskreditierung: Rauscher kündigt Hundert außerwelte / grosse / vnuerschempte / feiste / wolgemeste / erstunckene / Papistische Lügen [an - A.S.] Welche aller Narren Lugend / als des Eulenspiegels / Marcolphi / des Pfaffen vom Kalenbergs / Fortunati / Rollwagens / etc. weit vbertreffen [...]. ${ }^{62}$ Die Lügenden, so die Behauptung, überbieten jene unterhaltenden Erzählungen in Bezug auf ihren säkularen und fiktiven Gehalt und damit ihre Kurzweil. Die Lügenden werden also in den durch die Nennung der Romantitel(-figuren) aktualisierten Kontext erzählender Literatur mit trivial-komischem und oft derbem Inhalt gesetzt: Der Ulenspiegel, die

59 Vgl. u.a. Stenzel (1986) Rhetorischer Manichäismus, S. 7-9. In Anschluss an Stenzel vgl. auch Dieckmann (2005) Streiten, S. 45-51.

60 Entsprechend legt Luther in seiner in Anschluss an Augustinus entwickelten Lügentypologie im Nachwort zur Lügend Wert darauf, zwischen uneigentlichen Lügnern, dessen Lügen zur Unterhaltung dienen, und den „rechte[n] lügener[n] [zu unterscheiden - A.S], die mit ernst liegen und wissentlich die leute betriegen und beschedigen wollen." Luther (1537) Die Lügend, S. 61.

$61 \mathrm{Zu}$ hypertextuellen Verfahren vgl. Genette (1993) Palimpseste.

62 Rauscher (1562) Papistische Lügen I. 
Prosabearbeitung des mittellateinischen Dialogus Salomonis et Marcolfi - Salomon und Markolf -, Philipp Frankfurters in Reimpaarversen abgefasster Pfaffe von Kalenberg und Jörg Wickrams Rollwagenbüchlein gehören zu den Schwankromanen bzw. enthalten eine Sammlung von Schwankerzählungen, die im 16. Jahrhundert sehr populär sind. ${ }^{63}$ Ihre Geschichten spielen in sehr unterschiedlichen Milieus der Bauern, Handwerker, Kaufmänner und Kleriker, haben aber ihre schalkhaften Protagonisten gemeinsam. Deren intellektuelle Überlegenheit und Impertinenz stellen Ordnungen und Hierarchien in Frage, gleichwohl sind sie negative Helden: zuweilen grob, hässlich, vor allem moralisch nicht integer, da listig, skrupellos und auf den eigenen Vorteil bedacht. Solche Schalkfiguren, mit denen die Heiligen und Geistlichen hier konnotativ überschrieben werden, sind weder der imitatio noch der admiratio wert, sondern Negativexempel oder gar Beispiele für Sinnverweigerung. Mit ihnen wird das Angebot generiert, gemeinsam über ihre Erlebnisse, ihre Normbrüche und den durch sie verursachten Schaden zu lachen. Für die Lügenden werden "triviale Motive und Handlungselemente aus der derb-komischen Schwankliteratur, den kuriosen, oft auch makabren Sensationsberichten und der erotischen (italienischen) Novellistik" allerdings nicht übernommen, vielmehr werden mit ihnen solche in den legendarischen Erzählungen angelegten Motive ausgewählt und ausgestellt. ${ }^{64}$

Das Potential der ,Lügende' zur Textsortensignifizierung zeigt sich zweitens in den invektiven Anschlusskommunikationen von Seiten der Altgläubigen. Johannes Cochlaeus beispielsweise publiziert noch 1537 seinen Bericht der warheit / auff die vnwaren Lügend S. Johannis Chrysosto$m i,{ }^{65}$ mit dem er Luthers Schrift wiederum unter diskursiv umgekehrten Vorzeichen vermittels des Wahrheitsdiskurses als ,unwahr' zu deklarieren

63 Erfolgreich ist auch der Fortunatus, der als erster deutscher frei erfundener Prosaroman gehandelt wird. Er lässt sich aber nur bedingt in die Reihe der Schwankliteratur integrieren. Gleichwohl - und darauf kommt es hier an - wird die Handlung getragen von schwankhaften Erzählelementen und Märchenmotiven, die zwar in rationale Handlungszusammenhänge eingebettet werden, insgesamt aber eine Vielzahl von Unwahrscheinlichkeiten generieren.

64 Gegen Ecker (1993) Legende, S. 243.

65 Cochlaeus (1537) Bericht der warheit / auff die vnwaren Lügend S. Johannis Chrysostomi. versucht. ${ }^{66}$ Cochlaeus erkennt das herabsetzende und stigmatisierende Potential des Wortspiels, dem sofort widersprochen werden muss - bevor es sich zu einem gattungshaften Label entwickelt. Seine Wiederaufnahme der Bezeichnung ,Lügende' - nicht nur im Titel, sondern kontinuierlich auch im Bericht - bestätigt und reproduziert gleichwohl ihren Textklassifizierungswert, der sich mit der nachfolgenden Lügenden-Tradition mit Albers Alcoran und vor allem mit den Centurien Rauschers ausprägt, in denen der Begriff nicht nur in den Vorreden, sondern über den Titel hinaus in sämtlichen Paratexten zu finden ist. Auch Finck hat ab der zweiten Ausgabe seiner Neuauflage und Erweiterung der Rauscher-Centurien die ,Lügende' im Titelblatt aufgegriffen. ${ }^{67}$ Der Kolumnentitel bei Finck wird zudem ergänzt zu: "Hundert Papistischer / alter / vnhöfflicher Lügen". In den Zwischenüberschriften allerdings wird ,Exemplum' der ,Papistischen Lüge' vorgezogen - hier orientiert sich Finck an der zweiten Ausgabe von Rauscher, in der ,Exempla' verzeichnet werden; in den darauffolgenden Rauscher-Centurien genügen offenbar die thematischen Titel der Mirakelerzählungen samt Quellenverweisen, was für die Etablierung der Gattungsbezeichnung ,Lügende' sprechen könnte. Damit wird die gegeninvektive Strategie komplexer und vor allem auf Ebene der Gattungsmuster selbst angesiedelt, wie die Centurien des Franziskaners und Theologen Johannes Nas veranschaulichen. Mit seinen ,Gegenlügenden' kopiert Nas nämlich das invektive Verfahren der Lügenden und setzt es für die Bloßstellung und Herabsetzung der Reformatoren ein. Auf dem Titelblatt werden angekündigt: Das Antipapistisch eins vnd hundert. Außerleßner / gewiser / Evangelischer warhait / bey wölchen (als bey den früchten der Baum) die reyn lehr soll vnd muoß erkannt werden / Dann also spricht der Herr CHRISTVS, Ein yede pflantz so mein Vatter nit gepflantzt / muoß außgereüt werden. Die invektive Strategie beim Einsatz des Begriffs der Wahrheit kann hier als Ironie beschrieben werden, mit der ,Wahrheit' semantisch doppelt besetzt wird: Das Unzweifelhafte der, evangelischen Wahrheit' besteht nämlich darin, dass sie als Sünde erkannt und wie Unkraut vernichtet werden muss. Die

66 Die religiösen Diskurse über Wahrheit und Lüge sind eng miteinander verwoben; vgl. Fn. 57 und 58.

67 Finck (1614/18) Papistische Lügen, vgl. Fn. 50. 
Wahrheit, die der konfessionelle Gegner für sich beansprucht, habe er de facto nicht. ${ }^{68}$ Nach dieser Klarstellung ist es dann auch möglich, die vermeintlichen Wahrheiten als "gewiß erlogenen Euangelischen warhaiten" und gar im wortspielerischen Anschluss an die Lügende als "war lugen" zu bezeichnen. ${ }^{69}$

Sowohl die thematische als auch die rhematische Dimension des ersten Titelteils von Luthers Lügende bewegen sich nach der Taxonomie Genettes im Bereich der (Gattungs-)Konnotationen. Diese Konnotationen wiederum beruhen auf dem Wortspiel Lügende bzw. Lügenlegende als invektivem Sprachgebrauchsmuster. ${ }^{70}$ Bubenhofer hat im Rahmen seines Vorschlags zur Korpuslinguistik als Methode der Diskurs- und Kulturanalyse die Signifikanz von Sprachgebrauchsmustern als konstitutive Elemente von Kultur herausgestellt, weil sich in ihnen soziales Handeln manifestiere und sie somit als "Indikatoren für Diskurse" fungieren können. ${ }^{71}$ Bubenhofer greift unter anderem auf die soziolinguistischen Überlegungen Luckmanns und Bergmanns sowie Günthners und Knoblauchs zurück, die musterhaft verfestigten Formen alltagssprachlicher Kommunikation als, kommunikative Gattungen' bezeichnen. ${ }^{72}$ Als "kommunikative[r] Haus-

$68 \mathrm{Am}$ Ende der Vorrede appelliert Nas daher an seine Leser: „Frommer hertzlicher Leser / liß mit gedult vnd mit auffmerckung / die nachfolgenden artickel / der vermeinten / vnd fälschlich genannten Euangeliums warhait / ich wedt du werst der sachen weyter nachsinnen / vnd bey den losen früchten erkennen das der bawm kein nutz sey / vnd werdst dich von den Secten abwenden / vnd wider zuor einigkait kehren / vnd die Engel im Himel erfröwen / das wir dir von hertzen wünschen". Nas (1565) ,Evangelische Wahrheiten', Vorrede.

69 Nas (1565) ,Evangelische Wahrheiten', Register, das nach der Vorrede direkt anschließt. Durchgezählt werden die hundert Artikel allerdings als ,Evangelische Wahrheiten'. $70 \mathrm{Vgl}$. ferner ohne Bezug auf soziolinguistische Überlegungen zu Sprachgebrauchsmustern Sánchez (1999) Titel, hier S. 246: "Generell stützen sich Titel häufig auf volkstümlich etablierte Formeln, Gemeinplätze, Sprachhülsen, Slogans, geflügelte Worte des Alltags, oder banale Redewendungen, die dann aber mehr oder weniger abgeändert, doch immer noch erkennbar nachhallend, der neuen Aussage angepasst werden (und so ästhetisches Potential freisetzen). Parodierende Wiederverwertung von Altbekanntem."

71 Bubenhofer (2009) Sprachgebrauchsmuster, Titel und S. 6.

72 Vgl. u.a. Luckmann (1986) Grundformen; Bergmann (1987) Klatsch; Günthner/Knoblauch (1994) Forms are the Food of Faith; Günthner (1995) Gattungen in der sozialen halt" der Gesellschaft, bilden sie „das Herzstück dessen, was Kultur genannt werden kann. ${ }^{173}$

Diejenigen kommunikativen Vorgänge, die typisch wiederkehrend und deren regelmäßige Bewältigung von gesellschaftlicher Relevanz ist, bilden typische Muster aus, an denen sich Handelnde orientieren können. Kommunikative Gattungen bezeichnen diejenigen kommunikativen Prozesse, die sich gesellschaftlich verfestigt haben. ${ }^{74}$

Im Sinne eines stets prozesshaften Sprachhandelns können einzelne Sprachgebrauchsmuster Textsorten etablieren und determinieren sowie "als Bausteine von kommunikativen Gattungen gelesen werden". ${ }^{75}$ Dabei gilt es, die, eigentlichen' kommunikativen Gattungen als komplexe und stark formalisierte Formen von Sprachgebrauchsmustern mit ausgeprägtem handlungsverpflichtenden Charakter von den einfachen kommunikativen Mustern im Sinne von Günthner und Knoblauch zu unterscheiden. Bei der Wortverbindung Lügenlegende bzw. Lügende handelt es sich um ein Sprachgebrauchsmuster im Sinne Bubenhofers. ${ }^{76}$ Das Wortspiel kann sich insofern zu einem musterhaften Sprachgebrauch verfes-

Praxis; Günthner/Knoblauch (1996) Die Analyse kommunikativer Gattungen.

73 Günthner/Knoblauch (1994) Forms are the Food of Faith, S. 717, in Anschluss an Luckmann.

74 Günthner/Knoblauch (1994) Forms are the Food of Faith, S. 695f.

75 Vgl. Bubenhofer (2009) Sprachgebrauchsmuster, S. 45, hier S. 313. "Allerdings nehmen, wie gesagt, beileibe nicht alle kommunikativen Vorgänge die verfestigte Gestalt kommunikativer Gattungen an; einige sind gerade erst dabei, zu festen Gattungen zu gerinnen, wieder andere lösen sich in lockere, geregelte kommunikative Vorgänge auf." Günthner/Knoblauch (1994) Forms are the Food of Faith, S. 717.

76 „Ein Muster kann nur auf einer analytischen Ebene im Nachhinein festgestellt werden. Auf der Ebene des Sprachgebrauchs ist diese Musterfunktion für die Sprecherinnen und Sprecher kaum sichtbar. Mit ,musterhafter Sprachgebrauch' wird deshalb betont, dass anscheinend im untersuchten Sprachausschnitt immer wieder Instanzen einer bestimmten Phrase als Muster (als Vorbilder) für die Produktion weiterer Instanzen dienten. Im Nachhinein ist aber nicht mehr erkennbar, welche Instanzen je diese Musterfunktion übernahmen. Aber der Effekt dieser unzähligen Instanzen, die einerseits einem Muster folgten und andererseits Musterfunktion übernahmen, ist auf der Ebene der Analyse als Phänomen eines typischen, oder eben: musterhaften Sprachgebrauchs sichtbar." Bubenhofer (2009) Sprachgebrauchsmuster, S. 24. 
tigen, als es sich quasi über das Nomen Legende legt und dann, wenn das Wortspiel erfolgreich ist, im Wort Legende immer mittransportiert wird.

\begin{abstract}
Die Legende als Lügengeschichte zu bezeichnen, war schon seit Beginn der 1530er Jahre im Protestantismus geläufig. So verwendete Justus Jonas für seine Übersetzung von Philipp Melanchthons Apologia der [Augsburger] Confession (1531) den Begriff der Lügenlegende [...]. Mit Luthers 1537 erschienener Lügend von S. Johanne Chrysostomo setzte sich die Verballhornung von Legende in Lügende durch, die sehr viel griffiger und einprägsamer und deshalb für die Devianzproduktion sehr viel wirkungsvoller war. ${ }^{77}$
\end{abstract}

Griffigkeit und Einprägsamkeit sind Kennzeichen eines Sprachgebrauchsmusters, das hier in Bezug auf die ,lutherische Verhandlung' der Sache ,Legende' durch wiederholte Verwendung Erfolgswahrscheinlichkeit generiert. Wie zahlreiche Beispiele zeigen, wird der Begriff ,Lügende' "zum Gemeinplatz in der protestantischen Polemik."78 So werden ,Lügenden' auch in genuin anderen Kontexten als dem legendarischen Erzählen missbilligt - etwa in Luthers Auseinandersetzung mit der Donati Constantini (1537) oder in seiner Verlegung des Alcoran (1542). ${ }^{79}$ Caspar Goltwurms Kirchen Calender (1559) wiederum illustriert die Diffamierung der Legenden als Lügenden zur Abgrenzung von den eigenen Märtyrer- und Bekennerhistorien in nachlutherischer Zeit. ${ }^{80}$ Darüber hinaus ist die Invektive auch in protestantischen Schmähschriften, wie dem Binenkorb Des Heyl. Römischen Imenschwarms (1579) Johann Fischarts zu finden. ${ }^{81}$

Avanciert nun das (damit etablierte) Sprachgebrauchsmuster des Titels, Lügende' zur Gattungsbezeichnung, so schließen die weiteren Paratexte der Lügende an seine Funktion zur Herabsetzung der Legende an. Sprachpragmatisch gesehen verfestigen sie so das Muster, indem sie permanent darauf zurückgreifen. Textintern veranschaulicht diesen Prozess die erste Centurie Rauschers, bei der sich innerhalb der ersten drei Zwischenüberschriften - „Die erste Legenda

77 Münkler (2015) Legende/Lügende, S. 132.

78 Schenda (1974) Hieronymus Rauscher, S. 188.

79 Vgl. Luther (1537) Donatio Constantini, S. 74; Luther (1542) Verlegung des Alcoran, S. 391f.

80 Vgl. Goltwurm (1559) Kirchen Calender, Vorrede.

81 Vgl. Fischart (1579) Binenkorb, 2. Teil, 14. Kap.; 4. Teil, 4. Kap.; 5. Teil, 1. Kap.
/ Vom Teuffel vnd Prelaten", "Die ander Legenda. Von einem der gebeicht hat / nach dem jm der Kopff abgeschlagen ist" und "Die dritte Lugend. Von einem Blinden so der Kühe gehütet hat" die Transformation von der Legende zur Lügende vollzieht, um dann ab der siebten Mirakelerzählung auf den semantischen Gehalt verkürzt als "Bäpstisch Lüg" in Erscheinung zu treten und die Zählung am Seitenrand zu organisieren. ${ }^{82}$

Für die kommunikative Funktion des Wortspiels Lügende gehe ich darüber hinaus von einer kommunikativen Gattung - im weiteren Sinne des kommunikativen Musters - nach Günthner und Knoblauch aus, das sich dann auf die Gattungskonstitution überträgt. Denn erstens wird mit den Paratexten ein dialogisches Prinzip entworfen, das zur Auseinandersetzung der Rezipient:innen mit Inhalten der Legenden- und Mirakelerzählungen und ihren glaubensrelevanten Handlungsanweisungen anhält. Damit verweist sie nicht nur auf primär mündliche Interaktionssituationen - wie etwa auf Predigten, in denen Exempel angeführt werden -, sondern stellt mit der Lektüre selbst eine solche zwischen Mündlichkeit und Schriftlichkeit angesiedelte Interaktionssituation dar. Das zeigt sich besonders deutlich bei rhetorischen Fragen oder direkten Ansprachen an das Publikum in den Paratexten, ${ }^{83}$ grundsätzlich auch bei belehrenden Abschnitten vornehmlich in den Erinnerungen. Insgesamt aber evozieren die invektiven Paratexte den durch sie (affektiv) vorgeprägten Dialog mit den legendarischen Erzählungen auf Seiten der Rezipient:innen. ${ }^{84}$

Zweitens wird mit der Lügende eine schriftliche Textsorte etabliert, die starke Verfestigungen sowohl auf syntagmatischer Achse etwa mit der grundlegenden Folge von Erzählung und Erinnerung als auch in Hinblick auf ihre paradigmatische Textreihenbildung aufweist. Über eine komplexe Erzählform verfügt sie gleichwohl nicht. ${ }^{85}$ Zentral

82 Vgl. auch Holtzhauer (2020) Destruktion, Anm. 12 auf S. $95 f$.

83 Vgl. bspw. die Erinnerung zur 47. Lügend (Wie ein Vogel mit seinem gesang einen Mönich dreihundert Jar auff helt.) in Rauscher (1562) Papistische Lügen I: „Wie gefelt dir diser / meinstu nicht er werde auch ein Kleinat mit liegen gewunnen haben / vnnd hoch erhebt werden auff dem Luogenberg [...]."

84 Vgl. Fn. 113.

85 Über Grade und Qualitäten solcher Konventionalisierungen würde eine detaillierte Gattungsanalyse nach 
ist drittens, die Lügende als Lösungsangebot eines zentralen Problems religiöser Kommunikation in Zeiten der konfessionellen Ausdifferenzierung anzuerkennen: Wie gelingt es, die bedeutsame Rolle der Heiligen als Mittler zwischen Mensch und Gott herabzusetzen und den durch die altgläubige Kirche propagierten und funktionalisierten Heiligenkult lächerlich zu machen, ohne die Heiligen selbst grundlegend herabzuwürdigen?86 Die Heiligenverehrung war so stark in der religiösen Praxis verankert, dass theologische Argumente dabei nur wenig halfen, wie schon Luther im Nachwort seiner Lügend von St. Johanne Chrysostomo andeutet. ${ }^{87}$ Die Lügende lässt sich dahingehend als ein kommunikativer Gegenpol zu theologischen Aushandlungsprozessen innerhalb der Reformation begreifen, mit dem sie für das protestantische, Routineproblem' Entlastung schafft. ${ }^{88}$ Damit wird die Lügende zugleich Indikator für die Relevanz dieses kommunikativen ,Problems der Heiligenverehrung'. ${ }^{89}$

Günthner/Knoblauch (1994) Forms are the Food of Faith, Aufschluss geben. In Anschluss an Luckmann werden hier die analytischen Strukturebenen ,Binnenstruktur', ,Außenstruktur' und ,strukturelle Zwischenebene' differenziert. Eine solche Gattungsanalyse würde für das Thema des vorliegenden Aufsatzes allerdings zu weit führen.

86 Der heilige Franziskus (vgl. Rauscher-Beispiel im dritten Abschnitt) stellt dabei eine prominente Ausnahme dar, dessen Stigmata von den Protestanten wegen ihrer Gottähnlichkeit als besondere Anmaßung verstanden und energisch zurückgewiesen wurden.

87 Trotz aller Evidenz der Lügenhaftigkeit der altgläubigen Legende haben sie - so Luther - das Potential, Zweifel zu schüren. Vgl. Luther (1537) Die Lügend, S. 62f. Vgl. auch Luthers Vorrede in Alber(us) (1542) Der Barfuser Münche Eulenspiegel vnd Alcoran, in der er an den Leser appelliert, Gott darum zu bitten, nicht erneut in Finsternis und Blindheit zu geraten und nicht daran zu glauben, "das ein Ochse / Hund / Fisch / Schlange / Wurm / ja auch Zippeln vnd Knoblauch / Götter weren."

88 Vgl. Günthner/Knoblauch (1994) Forms are the Food of Faith, S. 693-696, S. 699f.

89 Vgl. Günthner/Knoblauch (1994) Forms are the Food of Faith, S. 700, S. 702, hier S. 716: Es "neigen vor allem jene kommunikativen Vorgänge zur Sedimentierung als Gattungen, die für die sozialen Akteure von bestimmter Relevanz sind. D.h. die kommunikativen Probleme, für die vorgeprägte, gattungsartige Lösungen im gesellschaftlichen Wissensvorrat einer Kultur existieren, sind für den Bestand einer Gesellschaft zentral. Deswegen können die Verfestigungen und Formalisierungen kommunikativer Vorgänge als Anzeichen für die gesellschaftliche Relevanz der durch sie gelösten kommunikativen Probleme angese-

\section{Paratext und Metagattung: Die Randglossen}

Neben der grundsätzlichen Problematisierung von Heiligenverehrung und Frömmigkeitspraxis rückt mit der Lügende konkret die Frage nach der religiösen Gattung Legende in den Fokus, nach ihren Möglichkeiten und Grenzen, im protestantischen Sinne angemessen von den Heiligen zu erzählen. Da die Lügende Gattungsreflexionen über die altgläubige Legende formuliert und beim Rezipienten auslösen will, ist sie als eine Metagattung, konkret als metalegendarisches Erzählen zu verstehen. ${ }^{90}$ Das Konzept der Metagattung geht zurück auf Wolf, der es im Rahmen seiner Systematisierung verschiedener Verfahren von literarischer und medialer Selbstreflexivität bzw. Metaisierung beschreibt. ${ }^{91}$ Unter Metaisierung versteht er ein "transgenerisches und transmediales Phänomen, das im Einziehen einer Metaebene in ein semiotisches System (ein Werk, eine Gattung oder ein Medium) besteht, von der aus Metareferenz erfolgt". ${ }^{92}$ Von einer Metagattung kann dann gesprochen werden, wenn „innerhalb eines semiotischen Systems von einer Metaebene Aussagen (z.B. Kommentare, Beschreibungen) über dieses System als solches oder über Teilaspekte desselben gemacht oder impliziert werden".93 Für diesen Vorgang bedarf es einerseits der Unterscheidung zwischen einer Subjekt- und einer Objektebene; vorausgesetzt wird also ein subjektiver Akt des Reflektierens über den literarischen Gegenstand dieser Reflexion. Andererseits muss dabei von einem Bewusstsein für die Artifizialität des entsprechenden Zeichensystems ausgegangen werden, das in der Regel mit dem Fiktionalitätscharakter eines Textes operiert fiktional sowohl im Sinne von, artifiziell-gemacht' (fictio-Aspekt) als auch im Sinne von ,potentiell erfunden' (fictum-Aspekt). ${ }^{94}$

Die protestantische Bloßstellung der Legenden als ,papistische Lügen' bedient ganz offensichtlich das, was Wolf als fictum-Aspekt bezeichnet. Der Vorwurf der Lüge bezieht sich sowohl auf

hen werden. Sie sind gleichsam die Knoten im dichten Netz der gesellschaftlichen Kommunikation."

$90 \mathrm{Vgl.} \mathrm{Sablotny} \mathrm{(2019)} \mathrm{Metalegende.}$

$91 \mathrm{Vgl.} \mathrm{Wolf} \mathrm{(2007)} \mathrm{Metaisierung.}$

92 Wolf (2007) Metaisierung, S. 38.

93 Wolf (2007) Metaisierung, S. 38.

94 Vgl. Wolf (2007) Metaisierung, S. 25. 
historisch nicht belegbare Ereignisse im Leben der Heiligen, welche die Legenden erzählen, als auch auf Heilsgewissheit versprechende Praktiken, die im Rahmen von christlicher imitatio und admiratio eingeübt werden (sollen). Dagegen werden Wahrscheinlichkeit und Historizität der Erzählungen als Qualitätsmerkmale propagiert, was zunächst der Diffamierung des legendarischen Erzählens von evangelischer Seite dient, im Zuge der Gattungsentwicklung aber in Teilen auch im katholischen Lager zumindest teilweise umgesetzt wird. ${ }^{95}$ Die Lügende wird aber darüber hinaus zur Metalegende, weil sie mit Blick auf Erzählelemente und Erzählweise die ,Gemachtheit' der altgläubigen Legenden (fictio-Aspekt) und ihre Funktionalisierung durch die römische Kirche bloßzulegen versucht. Die, papistischen Lügen' seien - so Luther in seinem Nachwort nicht nur "ertichtet", sondern es sei auch „alles drinnen dahin gericht", Messen, Fegefeuer etc. zu propagieren. ${ }^{96}$ So verdeutlicht die Glosse "Je, das mus ja war sein, wer kundts erdencken!", ${ }^{97}$ welche den kräftigen Windstoß kommentiert (s.o.), nicht nur die Unwahrscheinlichkeit der Handlung im Sinne einer fehlenden Authentizität der Erzählung (fictum-Aspekt). „Erdencken" rekurriert zudem auf den Vorgang des Erfindens, wodurch der Legenden-Autor bzw. -Verantwortliche zum Legenden- „Poeten" wird. ${ }^{98}$ Invektiv ist nicht allein der Fiktionsvorwurf, sondern die damit verknüpfte Fiktionalisierung der altgläubigen Legenden zu zumindest für die Protestant:innen - unterhaltsamem "schimpff und schertz". ${ }^{99}$

95 Vgl. die Herausbildung von Bekennerhistorien und auch Historien von Märtyrern auf protestantischer Seite, welche die Heiligen als Glaubensvorbilder und Vermittler von Trost, nicht aber als Fürbitter in Szene setzen. Wie die Bezeichnungen Exempla oder Historien ankündigen, erheben diese, gereinigten' Legenden im Gegensatz zu denen der Altgläubigen den Anspruch auf Wahrhaftigkeit und Exemplarizität. Auf katholischer Seite evoziert die virulente Legendenkritik einen neuen, historisch-kritischen Legendentyp, der schließlich in das groß angelegte hagiographische Projekt der Bollandisten mündet - der erste Band der Acta Sanctorum erscheint 1643, der letzte 1940. Vgl. Münkler (2015) Legende/Lügende, S. 138-142, dort mit Quellen.

96 Luther (1537) Die Lügend, S. 62.

97 Luther (1537) Die Lügend, S. 57.

98 Luther (1537) Die Lügend, S. 46. Für eine ausführliche Darstellung vgl. Sablotny (2019) Metalegende, v.a. S. 180-188. 99 Luther (1537) Die Lügend, S. 61.
Diese funktionale Kopplung von Gattungsreflexion (Gattungskritik gegenüber der Legende) und Gattungstransformation (von der Legende zur Lügende und zur Unterhaltungsliteratur) wird durch die Paratextualisierung der Legenden- und Mirakelerzählungen geleistet. ${ }^{100}$ Demnach wären die invektiven Paratexte als bloßes ,Beiwerk' der Lügende ohne Zweifel fehlbestimmt. Vielmehr werden sie zu den konstitutiven Elementen der Metagattung. ${ }^{101}$ Das gilt vorrangig für die unmittelbaren Kommentierungen der abgedruckten Erzähltexte, das heißt für die Randglossen sowie für die ,Erinnerungen' bei den Rauscher-Centurien, die im nahen räumlichen Umfeld des jeweiligen Einzeltextes stehen. Mit der Differenzierung zwischen Marginalien und ,Erinnerungen' wird bei Rauscher eine hierarchische Abstufung der Paratextelemente suggeriert, da Letztere eben nicht an den Rändern, sondern unmittelbar nach den Mirakelerzählungen platziert werden. ${ }^{102}$ Als vergleichsweise ausführliche Kommentierungen mit - durch die Überschrift noch verstärkter - textueller Eigenständigkeit verschwimmen hier die typologischen Grenzen von Paratextualität und Metatextualität insofern, als die ,Erinnerungen' auf den jeweils zuvor abgedruckten Basistext kritisch-reflektierend Bezug nehmen. ${ }^{103}$ Gegen die Zuordnung der ,Erinnerungen' zu den Metatexten sprechen ihre Genese aus den Kontextglossen und ihre kopräsente, rezeptionssteuernde

100 Das Verhältnis von Paratext(ualität) und Fiktion(alität) im Kontext literarischer Selbstreflexivität ist Schwerpunkt der Studie von Kuhn (2018) Wahre Geschichten, allerdings mit historischem Fokus auf das 18. und 20. Jahrhundert. „Fiktion und Paratext sind [...] parallele und zugleich wechselseitig verschränkte Phänomene: Ist der Paratext Teil der Fiktion, so ist er Teil des Werkes selbst, ist er letzteres nicht, so kann er nicht Teil der Fiktion sein." Ebd., S. 29f. Im Sinne der modernen Romantexte wird der ,eigentliche Text' in der Regel mit Fiktion gleichgesetzt. Dagegen wird die Legende als Lügende mit ihren Paratexten erst zur Fiktion gemacht.

101 Mit Bezug auf Stanitzek (2004) Texte, Paratexte, S. 8, siehe auch Schmidt (2008) Pluralisierung, S. 228: Für Thomas Mores literarischen Dialoge sei der Paratext "geradezu der bestimmende Faktor für die Identität des Textes selbst - ,nicht bloß eine Zone des Übergangs, sondern der Transaktion', wie Genette auch selbst bereits erkannt hat." Zur Bedeutung des Rands für die Konstitution des Textes vgl. auch Menke (2015) Text-Oberfläche, S. $130 f$.

102 Zur visuellen Unterscheidung von glosse und textus vgl. Menke (2015) Text-Oberfläche, S. $135 f$.

103 Vgl. Genette (1993) Palimpseste, S. 13. 
Zurichtung der legendarischen Erzählungen. Bei ihnen handelt sich daher vielmehr um eine Form der (para-)textuellen Verselbständigung, die als strategische Einschreibung des Invektiven in die altgläubige Mirakelerzählung lesbar wird. Das Vorgehen ist funktional vergleichbar mit der textinternen Transformation der Zwischentitel gebenden ,Legende' zur ,Lügende' bzw. ,Lüge' in der ersten Rauscher-Centurie. ${ }^{104}$

Die Randglossen und ,Erinnerungen' gehen weit über die Eigenheiten und Funktionen der ,Anmerkungen', wie sie Genette erläutert, ${ }^{105}$ hinaus. Im Grunde stehen sie quer zu ihnen: Denn sie sind gerade nicht nur durch den beiläufigen Charakter einer Ergänzung oder Abschweifung bestimmt und besitzen daher auch nicht nur den "Status einer fakultativen Lektüre". ${ }^{106}$ Zudem hat Genette vorrangig auktoriale Anmerkungen im Sinn; die von diesen zu unterscheidenden allographen Anmerkungen engt er ein auf "fast zwangsläufig[e]" verlegerische Anmerkungen, die noch dazu gar "nicht unter die Definition des Paratextes" fallen würden. ${ }^{107}$ Für die nur im weiteren Sinne als, authentisch allographe' Anmerkungen zu verstehenden invektiven Paratexte der Lügende gäbe es hier keinen Platz. ${ }^{108}$ Auch Genettes grundsätzliche Differenzierung „zwischen Anmerkungen in Zusammenhang mit diskursiven Texten (geschichtliche Werke, Essays usw.) und den [...] Anmerkungen, die Werke der narrativen oder dramatischen Fiktion oder der lyrischen Dichtung zieren oder [...] entstellen", ${ }^{109}$ lässt sich für die Legenden/Lügenden nicht ohne Weiteres übernehmen. Das liegt vor allem am Textstatus der Legende, der mit der Differenz von Faktizität und Fiktionalität nicht einzuholen ist. ${ }^{110}$ Zwar besteht mit ihr der Anspruch, Glaubenswahrheiten zu vermitteln und zu verstetigen. Sie lässt aber durchaus "narrative Elemente zu, die aus fiktionaler Erzähltradition stammen, in der Legende jedoch sofort in den Status von, geschichtlicher

$104 \mathrm{Vgl}$. vierten Abschnitt.

105 Vgl. Genette (2001) Paratexte, S. 304-327.

106 Vgl. Genette (2001) Paratexte, S. 308f., hier S. 308.

107 Genette (2001) Paratexte, S. 321.

108 Zur Differenzierung von allographen und autographen Marginalien vgl. Gioriato (2008) Prolegomena zur Marginalie.

109 Genette (2001) Paratexte, S. 309.

110 Vgl. Glauch (2014) Fiktionalität, S. 96, S. 111, S. 124.
Wahrheit' überführt werden".111 In diesem Sinne konstatiert Glauch für das Mittelalter, aber auch noch für das 17. und 18. Jahrhundert "eine grundsätzliche Übergängigkeit von nichtfiktionalen und fiktionalen Erzählformen und -registern", insbesondere "zwischen Historiographie und Epik sowie zwischen religiösem und weltlichem Erzählen."112 Der ,Witz' und damit das Invektive der Lügende wiederum liegt darin, die latente Ambiguität legendarischen Erzählens durch die Fiktionalisierung der Legenden zu vereindeutigen. Die protestantische Lektüre kommentiert verlachend die ,Gemachtheit' der Legenden.

Im Modell der Metagattung ist die Paratextualisierung also das zentrale Metaisierungsverfahren in der Lügende. Mit ihm gelingt das Einziehen einer Metaebene, d.h. die Trennung zwischen dem Objekt ,Legende' und dem reflektierenden Subjekt, das einerseits mit dem jeweiligen Herausgeber der Lügenden identifiziert werden kann, andererseits aber die Leser:innen adressiert. Indem die Paratexte als Interpretationssteuerung fungieren und in diesem Sinne eine die Legenden herabsetzende Lektürehaltung einüben sollen, ${ }^{113}$ werden die Rezipient:innen der Lügenden zum kritisch reflektierenden Subjekt gemacht.

Weil bei der Lügende die metaisierenden Paratextelemente zu einem gattungskonstituierenden Merkmal avancieren, ist deren textuelle Zugehörigkeit nicht eindeutig bestimmbar. ${ }^{114}$ Diese Beobachtung führt zurück auf den nicht nur bei Genettes Kritikern, ${ }^{115}$ sondern von Genette selbst problematisierten Status des Paratextes und seine wenig trennscharfe Unterscheidung zwischen Text, Paratext und Kontext: Auch jenseits der Differenzierung zwischen Peritexten

111 Vollmann (2002) Erlaubte Fiktionalität, S. 72.

112 Glauch (2014) Fiktionalität, S. 112.

113 „Das übliche Gegenüber von Autor und Rezipient tritt hier zurück, die späteren Leser schauen dem ersten Leser bei seinem Glossieren über die Schulter. So betrachtet enthalten die Glossen einen Appell ans Publikum, sich seine eigenen Glossen auf den Text zu finden". Schnyder (1979) Legendenpolemik, S. $129 f$.

114 Auf das hohe Metaisierungspotential von Paratexten hat schon Struth hingewiesen. Ihr Fokus auf englischsprachige postmoderne Metaautobiographien mag erklären, dass sie gleichwohl die theoretische Trennung des Paratextes vom eigentlichen ,Kerntext' voraussetzt. Vgl. Struth (2014) Metagattungen, S. 271f. sowie Struth (2016) Die Metaautobiographie, S. 84-98.

$115 \mathrm{Vgl.}$ Assmann (2018) Zur Unterscheidung, S. 37-39. 
und Epitexten spricht er von Nähe- bzw. Distanzgraden der Paratexte zu Text und Kontext. ${ }^{116}$ Die ,inneren' und ,äußeren' Grenzen des Paratextes sind variabel und durchlässig, weil die grundsätzliche pragmatische Funktion des Paratextes eine zwischen Text und ,Welt' vermittelnde ist. ${ }^{117}$ Diese

116 Die Problematisierung des Genette'schen Paratextkonzepts für Texte des Manuskriptzeitalters bei Cooper (2015) scheint mir auf eine eher simplifizierende Vorannahme von einer scharfen Trennung von Text und Paratext zu beruhen (vgl. ebd., S. 45), die ich bei Genette so nicht sehe. Die Betonung des (nicht nur) mittelalterlichen Paratextes als eine dynamische Größe, die sich aus Prozessen von (Re-)Kontextualisierungen von Texten ergibt, ist hingegen ganz richtig. Daraus ergeben sich in der Tat Herausforderungen für wissenschaftliche Texteditionen. Vgl. ebd., S. 46. Für das Verständnis mittelalterlicher Paratexte hat Kragl (2016) Die (Un-)Sichtbarkeit, die unfeste Grenze von Text und Peritext exemplarisch herausgearbeitet und unterscheidet ,unsichtbare' von ,sichtbaren' Elementen im handschriftlichen Buch. Diese funktional geleitete Differenzierung erweitert das paratextuelle Feld mittelalterlicher Literatur analytisch gewinnbringend. Vgl. schon Münkler (2000) Erfahrung, S. 240-266, hier S. 241: „In der Tat sind Paratext und Text in der handschriftlichen Überlieferung nicht in derselben Weise voneinander abgrenzbar wie in gedruckten Büchern. Die Grenzen zwischen beiden sind häufig verwischt, weil sie weniger deutlich durch eine festgelegte Anordnung, durch Seitenwechsel oder das Schriftbild voneinander abgetrennt sind. Dennoch kennt die handschriftliche Überlieferung zweifellos Paratexte in der Form von Rubriken, incipites und explicites, Prologen und Epilogen, Anfangsinitialen, Autorenbildern oder anderen vorangestellten Illustrationen, die den Lesehorizont des Textes prägen." Vgl. im Kontext des Parzival-Prologs auch Sablotny (2010) Zeit und âventiure, S. 38f. Zur Problematisierung des Paratextkonzepts im Kontext von Dramenprologen hingegen vgl. Wolf (2008) Prologe. Wolf entwickelt hier das alternative und ergänzende Modell der literarischen Rahmung: "als Alternative insofern, als es an die Stelle von ,Paratext' als Dachbegriff für die verhandelten Phänomene treten kann; als Ergänzung insoweit, als ein klar konturierter (auf verbale, werkinterne Texte beschränkter) Paratextbegriff unter diesem Dach weiterhin seinen Ort haben könnte - als eine wichtige Form der Rahmung neben anderen." Ebd., S. 95.

$\mathbf{1 1 7}$ Mit Fokus auf Vorworterzählungen Jean Pauls können sich nach Wirth Paratexte - und hier besonders Vorworte - selbst "als Übergangszone in Szene setzen [...], in der die Grenzen zwischen all dem, was fiktiver Text ist und all dem, was nicht fiktiver Text ist, verhandelt werden." Wirth (2009) Paratext, S. 167. Wirth beschreibt diesen Vorgang als ein "paratextuelle[s] travelling" (ebd.), mit dem sowohl Bewegungen im ,Vorraum' des Textes als auch Bewegungen zwischen Text und Paratext gemeint sind. Darüber hinaus hätten solche zu beobachtende und in den Vorworten ausgestellte ,Wegbeschreibungen' eine performative Dimension: Paratexte seien „Übergangszonen, die
Vermittlungsleistung ist entscheidend für die Ausprägung der Metagattung, die dazu dient, die ihr zugrundeliegende Gattung zu destruieren. Für die als ,papistische Lügentexte' enttarnten Legenden sind die Paratextelemente daher konstitutiv. ${ }^{118}$

\section{Conclusio: Invektive Paratexte als sekundäre Formen religiöser Kommunikation}

Greifen Text und Paratext, wie ich an der paratextuellen Überschreibung der Legende und ihrer Transformation zur Lügende gezeigt habe, funktional ineinander über, so wird das Transitorische bzw. Transaktive und Diskursive des Paratextes in die Gattung selbst implementiert. Die Paratextualisierung erweist sich als ein wirkmächtiger Modus des Invektiven, weil sie invektive Anschlusskommunikationen initiiert und potenziert: In der zur Lügende transformierten Legendenerzählung scheinen auch nicht glossierte Textelemente im Sog des Invektiven zu stehen. Das könnte erklären, warum das Paratextualisierungspotential offenbar nicht gänzlich ausgeschöpft wird. Luthers Marginalglossen beispielsweise kommentieren Erzählelemente vom Beginn bis zum zweiten Drittel der ChrysostomusLegende; sie reduzieren sich dann auf zwei Anmerkungen bis zum Ende. Gleichwohl fungieren sie als Auslöser weiterer Invektiven. In den Drucken der Rauscher-Centurien finden sich handschriftliche, d.h. sekundäre Glossen von Lesern, die das dynamisierende Potential der Paratextelemente vor Augen führen. Sie verweisen zugleich auf die dann textextern zu verortenden Effekte, zu denen auf der einen Seite die protestantische, auf der anderen Seite die ihr gegenüberstehende katholische Lektüre- und Schmähgemeinschaft gehören. ${ }^{119}$

durch eine doppelte Geste - die Geste der Grenzziehung und die Geste der Grenzüberschreitung - eine Grenze zwischen Außenwelt und Textwelt schaffen." Ebd., S. 175. Die Marginalie fokussierend spricht auch Giuriato (2008) Prolegomena zur Marginalie, S. 177, vom Paratext als einem "liminalen Phänomen", das "die Grenze zum Text ebenso regeln wie auflösen" kann.

118 Eine Zuordnung der Lügenden zu 'parasitären' Legendenparodien (vgl. Ecker [1996] Art. Legende, S. 858) verdeckt das innovative und kommunikative Gattungspotential der Lügende.

119 Vgl. die 1562 in Neuburg an der Donau gedruckte erste Centurie Rauschers (VD16 ZV 17431). Zu den 
Die Paratextualisierung der Legenden- und Mirakelerzählungen schreibt zudem auf Ebene der Gattungsdynamik invektiv-kommunikative Prozesse fort: Die protestantischen und katholischen Transformationen der Legende in Lügende, 'Gegenlügende' (Johannes Nas' Evangelische Wahrheiten), Historie (insbesondere Ludwig Rabus' protestantisches Martyrologium) und historisch-kritische Legende (die Acta Sanctorum) samt ihren Vor- und Nachworten lassen sich als ein Netz invektiver Anschlusskommunikationen beschreiben. ${ }^{120}$ Außerhalb dieser Gattungsgrenzen lagern sich daran diskursive Gattungen wie Streitschriften, Predigten, Gegenberichte beispielsweise von Johannes Cochlaeus, Friedrich Staphylus oder Martin Eisengrein an, die schließlich die Proliferation der Paratextualisierungen darstellen. Mit innen wird sowohl direkt als auch indirekt über die Gattung Legende reflektiert; mit ihnen wird ausgehandelt, wie vom Heiligen erzählt werden könne.

Im Sinne dieser funktionalen Dimension möchte ich Paratexte hier als Organisatoren bzw. sekundäre Formen von religiöser Kommunikation auffassen. ${ }^{121}$ Luhmann hat seine Überlegungen zu den primären und sekundären Kommunikationsformen zwar im Kontext des Kunstsystems entwickelt, sie lassen sich aber auf das Religions-

handschriftlichen Glossen Holtzhauer (2020) Destruktion, Anm. 17 auf S. 97: „[E]s könnte sehr erhellend sein, diese Kommentare einmal zu sammeln und auszuwerten. Vielversprechend erscheinen [...] jene Bemerkungen in den protestantischen Schriften, die nachgewiesenermaßen von katholischen Lesern stammen, könnten sie doch die ,dialektische[] Abhängigkeit' der katholischen und protestantischen Legendensammlungen (Schenda 1970, S. 47) tiefergehend ergründen helfen."

120 Zum gattungshistorischen bzw. gattungsdynamischen Zusammenhang der Lügende vgl. Sablotny (2019) Metalegende, S. 166-169; Münkler (2015) Legende/Lügende, S. 138-142; Münkler (2011) Narrative Ambiguität, S. 134-142; Münkler (2008) Sündhaftigkeit, S. 40-46; Ziegeler (1999) Wahrheiten; Ecker (1993) Legende, v.a. S. 238-243; Schnyder (1979) Legendenpolemik, S. 138f.; Schenda (1972) Hieronymus Rauscher, S. 187-199; Schenda (1970) Legendenpolemik, S. S. 40-44; Hieber (1970) Legende.

121 In Anschluss an Stanitzek (2004) Texte, S. 12, bzw. Assmann (2018) Zur Unterscheidung, der sich mit Fokus auf das ausdifferenzierte Kunst- bzw. Literatursystem der Moderne an der vermeintlichen Autorzentriertheit Genettes abarbeitet. Zur religiösen Kommunikation vgl. Luhmann (2000) Religion der Gesellschaft. system übertragen - insbesondere, um die in die Metagattung implementierte Öffnung zum Diskursiven zu konturieren. Dort sind sekundäre Formen Operationen der Beobachtung von primären Formen der Kunst bzw. Literatur. Sie machen den an sich unwahrscheinlichen Erfolg, Kunstoder Literaturwerke als solche wahrzunehmen, wahrscheinlicher. ${ }^{122}$ Hier erfüllen die Paratexte der Lügende als sekundäre Formen religiöser Kommunikation allerdings gegenteilige Funktion: Mit ihnen soll der kommunikative Erfolg der altgläubigen Legenden- und Mirakelerzählungen abgestellt, ihre religiöse Wahrheit im apotropäischen Sinne geradezu ausgetrieben werden ${ }^{123}$ - gleichsam, um die protestantischen Historien an ihrer Stelle zu etablieren. Die liminale Phase des Religionssystems im Zuge der Reformation, genauer gesagt die liminale Phase seiner Programme, ${ }^{124}$ wie das der Heiligenverehrung, macht die Bedeutsamkeit sekundärer Formen religiöser Kommunikation evident. ${ }^{125}$ Sie stehen den pragmatischen primären Formen des Religionssystems wie den Legenden näher als die theologisch-argumentativ geführten Textgattungen. Für den protestantisch abzulehnenden Heiligenkult etabliert die Lügende den invektiv geführten Kommunikationsmodus offenbar so stark, dass er die Grenzen des primär konfessionellen Diskurses überschreitet - beispielsweise bei der paratextuellen Rahmung der Brandan-Legende in Gabriel Rollenhagens Vier Bücher Wunderbarlicher biß daher vnerhörter / vnd vngleuplicher Indianischer Reysen / durch

122 Vgl. Luhmann (2002) Kunst der Gesellschaft; Luhmann (2008) Medium der Kunst.

123 Vgl. Fn. 7.

124 Zur Deutung der Reformation als rituellem Prozess mit Kennzeichen von Liminalität im Sinne von Arnold van Gennep bzw. Victor Turner vgl. u.a. Pfrunder (1989) Pfaffen, S. 253f., oder von Bernuth (2009) Wunder, S. 74.

125 "Die Selbstregulierung und Selbstkontrolle des Systems ergeben sich [...] auf der Ebene der Programme, die die Beobachtung der Operationen durch das System selbst leiten (aufgrund anderer Unterscheidungen als denjenigen, an denen diese Operationen sich orientieren). Die Programme stellen die Bedingungen fest, die für die Realisierbarkeit einer bestimmten Operation gegeben sein müssen. Sie bestimmen zum Beispiel, daß die Zuschreibung des positiven Codewertes nur unter gewissen Bedingungen korrekt ist." Esposito (1997) Art. Programme, S. 139. 
die Lufft / Wasser / Land / Helle / Paradiß / vnd den Himmel (1608). ${ }^{126}$

Begreift man die Lügende als diese in ein Gattungsformat gebrachte Übergängigkeit, als ein Kondensat der invektiv geführten Aushandlungsphase im 16. Jahrhundert und noch zu Beginn des 17. Jahrhunderts, so wird erstens ihre außerordentliche Signifikanz während der religiösen Ausdifferenzierung, aber auch zweitens ihre damit zusammenhängende gattungshistorische Begrenztheit auf diese Zeit des Übergangs deutlich. Die Lügende ist daher im doppelten Sinne ein Schwellenphänomen.

\section{Literaturverzeichnis}

\section{Quellen}

Alber(us), Erasmus (1542): Der Barfuser Münche Eulenspiegel vnd Alcoran. Wittenberg: Hans Lufft (VD16 A 1477).

Brand, Margit/Jung, Bettina/Williams-Krapp, Werner (Hg.) (2004): Der Heiligen Leben. Bd. 2: Der Winterteil. Tübingen: Max Niemeyer.

Cochlaeus, Johannes (1537): Bericht der warheit / auff die vnwaren Lügend S. Johannis Chrysostomi [...]. Leipzig: Nikolaus Wolrab (VD16 C 4268).

Finck, Caspar (1614/18): [...] Papistische Unwahrheiten/ Lügen [...]. Gießen et al.: Nicolaus Hampel et al. (VD17 1:076768U et al.).

Fischart, Johann (1579): Binenkorb Des Heyl. Römischen Imenschwarms / seiner Hummelszellen (oder Himmelszellen) Hurrnaußnäster / Brämengeschwürm vnd Wäspengetöß [...]. Straßburg: Bernhard Jobin.

Goltwurm, Caspar (1559): Kirchen Calender. Ein Christlich vnd nützlich Buoch [...]. Frankfurt a.M.: Christian Egenolff d. Ä. (VD16 G 2589).

Luther, Martin (1519): Bekräftigung aller Artikel Martin Luthers, die durch die Bulle Leo X. neuerlich verdammt worden sind. In: Martin Luthers Werke. Kritische Gesamtausgabe. Abteilung 1: Schriften, Bd. 1-56. Weimar: Böhlau 1883-1929, Bd. 7 [= WA 7], S. $94-151$.

Luther, Martin (1522): Bulla coenae domini, das ist, die Bulla vom Abendfressen des allerheiligsten Herrn, des Papstes, verdeutscht durch Martin Luther. In: WA 8, S. $688-720$.

Luther, Martin (1542): Verlegung des Alcoran Bruder Richardi, Prediger Ordens. In: WA 53, S. 261-396.

126 Vgl. Koch (2017) Das Abenteuer, S. 56-62, hier S. 62: "Die Brandan-Legende steht weniger im Dienst protestantischer Kritik als vielmehr die protestantische Kritik im Dienst einer abenteuerlichen Lektüre von Brandans Meerfahrt zum Paradies."
Luther, Martin (1537): Die Lügend von St. Johanne Chrysostomo. In: WA 50, S. 48-64.

Nas, Johannes (1565): Das Antipapistisch eins vnd hundert. Außerlesner / gewiser / Evangelischer wahrhait [...], Ingolstadt: Weißenhorn, Alexander II. und Weißenhorn, Samuel (VD16 N 96).

Rauscher, Hieronymus (1562-64): [...] Papistische Lügen [...]. Regensburg et al.: Heinrich Geißler et al. (VD16 R 399; VD16 ZV 17431; VD16 R 400; VD16 R 403 et al.). Vergerio, Pietro Paolo (1556): De Gregorio Papa [...]. Königsberg: Hans Daubmann (VD16 G 3130).

\section{Forschungsliteratur}

Ammon, Frieder von (2007) "Quis dubitat de illo?" The ,Staging' of Religious Pluralization through Paratexts. In: Höfele, Andreas/Laqué, Stephan /Ruge, Enno et al. (Hg.): Representing Religious Pluralization in Early Modern Europe. Münster: LIT, S. 279-299.

Ammon, Frieder von (2006) „Bevor wir Dich hören, Heiligster." Die Paratextualisierung der päpstlichen Autorität in Ulrich von Huttens Edition der Bulle Exsurge Domine. In: Sonderforschungsbereich 573. Pluralisierung und Autorität in der Frühen Neuzeit. 15.-17. Jahrhundert. Mitteilungen 1, S. 31-38. DOI: $10.5282 /$ ubm/epub.74904 (letzter Zugriff: 07.11.2021).

Ammon, Frieder von/Vögel, Herfried (2008): Die Pluralisierung des Paratextes in der Frühen Neuzeit. Einleitung. In: Dies. (Hg.): Die Pluralisierung des Paratextes in der Frühen Neuzeit. Theorie, Formen, Funktionen. Berlin: LIT Verlag, S. VII-XIX.

Assmann, David-Christopher (2018): Zur Unterscheidung von primären und sekundären literarischen Formen. In: Gerstenbräun-Krug, Martin/Reinhard, Nadja (Hg.): Paratextuelle Politik und Praxis. Interdependenzen von Werk und Autorschaft. Wien: Böhlau, S. 37-52.

Behringer, Wolfgang (2005-12): Art. Lüge. In: Jaeger, Friedrich (Hg.): Enzyklopädie der Neuzeit Online 7. URL: http://dx.doi.org/10.1163/2352-0248_edn_ COM_305395 (letzter Zugriff: 07.11.2021).

von Bernuth, Ruth (2009): Wunder, Spott und Prophetie. Natürliche Narrheit in den ,Historien von Claus Narren'. Tübingen: Max Niemeyer.

Bergmann, Jörg (1987): Klatsch. Zur Sozialform der diskreten Indiskretion. Berlin: De Gruyter.

Bubenhofer, Noah (2009): Sprachgebrauchsmuster. Korpuslinguistik als Methode der Diskurs- und Kulturanalyse. Berlin/New York: De Gruyter.

Carmassi, Patrizia/Heitzmann, Christian: Einleitung. In: Dies. (Hg.): Marginalien in Bild und Text. Essays zu mittelalterlichen Handschriften. Wiesbaden: Harrassowitz Verlag, S. 7-18.

Cooper, Charlotte E. (2015): What ist Medieval Paratext? In: Marginalia 19, S. 37-50.

Dieckmann, Walther (2005): Streiten über das Streiten. Normative Grundlagen polemischer Metakommunikation. Tübingen: Max Niemeyer. 
Dietzsch, Steffen (2001): Lüge. Umriß einer Begriffsgeschichte. In: Röttgers, Kurt/Schmitz-Emans, Monika: „Dichter lügen". Essen: Die blaue Eule, S. 15-35.

Ecker, Hans-Peter (1993): Die Legende. Kulturanthropologische Annäherung an eine literarische Gattung. Stuttgart/Weimar: J.B. Metzler.

Ecker, Hans-Peter (1996): Art. Legende. In: Enzyklopädie des Märchens 8, Sp. 855-868.

Ellerbrock, Dagmar/Koch, Lars/Müller-Mall, Sabine et al. (2017): Invektivität - Perspektiven eines neuen Forschungsprogramms in den Kultur- und Sozialwissenschaften. In: Kulturwissenschaftliche Zeitschrift 2/1, S. 2-24.

Esposito, Elena (1997): Art. Programm. In: Baraldi Claudio/Corsi, Giancarlo/Esposito, Elena: GLU. Glossar zu Niklas Luhmanns Theorie sozialer Systeme. Frankfurt am Main: Suhrkamp, S. 139-141.

Genette, Gérard (1993): Palimpseste. Die Literatur auf zweiter Stufe. Aus dem Französischen von Wolfram Bayer und Dieter Hornig. Frankfurt am Main: Suhrkamp [Paris 1982].

Genette, Gérard (2001): Paratexte. Das Buch vom Beiwerk des Buches. Mit einem Vorwort von Harald Weinrich. Aus dem Französischen von Dieter Hornig. Frankfurt am Main: Suhrkamp [Paris 1987].

Giuriato, Davide (2008): Prolegomena zur Marginalie. In: Ders./Stingelin, Martin/Zanetti, Sandro (Hg.): "Schreiben heißt: sich selber lesen". Schreibszenen als Selbstlektüren. München: Wilhelm Fink, S. 177-198.

Glauch, Sonja (2014): Fiktionalität im Mittelalter; revisited. In: Poetica 46, S. 85-139.

Großhans, Hans-Peter/Sparn, Walter (2005-12): Art. Wahrheit. In: Jaeger, Friedrich (Hg.): Enzyklopädie der Neuzeit Online 14. URL: http:// dx.doi.org/10.1163/2352-0248_edn_COM_376630 (letzter Zugriff: 07.11.2021).

Günthner, Susanne (1995): Gattungen in der sozialen Praxis. In: Deutsche Sprache 3, S. 193-218.

Günthner, Susanne/Knoblauch, Hubert (1994): „Forms are the Food of Faith". Gattungen als Muster kommunikativen Handelns. In: Kölner Zeitschrift für Soziologie und Sozialpsychologie 46, S. 693-723.

Günthner, Susanne/Knoblauch, Hubert (1996): Die Analyse kommunikativer Gattungen in Alltagsinteraktionen. In: Michaelis, Susanne/Tophinke, Doris (Hg.): Texte - Konstitution, Verarbeitung, Typik. München: LINCOM Europa, S. 35-57.

Hamm, Joachim (2017): Zu Paratextualität und Intermedialität in Sebastian Brants Vergilius pictus (Straßburg 1502). In: Robert, Jörg (Hg.): Intermedialität in der Frühen Neuzeit. Formen, Funktionen, Konzepte. Berlin/ Boston/New York: De Gruyter, S. 236-259.

Hempfer, Klaus W. (1973): Gattungstheorie. Information und Synthese. München: Wilhelm Fink 1973.

Herms, Eilert (2003): Art. Wahrheit/Wahrhaftigkeit. In: Theologische Realenzyklopädie 35, S. 337-378.

Hieber, Wolfgang (1970): Legende, protestantische Bekennerhistorie, Legendenhistorie. Studien zur literarischen Gestaltung der Heiligenthematik im Zeitalter der Glaubenskämpfe. Diss. Würzburg.
Holtzhauer, Sebastian (2020): Die Destruktion der Wunderzeit in Hieronymus Rauschers ,Papistischen Lügen' (1562). In: Beiträge zur mediävistischen Erzählforschung 3, S. 66-109.

Jaser, Christian (2016): Ostensio exclusionis. Die päpstliche Generalexkommunikation zwischen kirchenrechtlicher Innovation und zeremoniellem Handeln. In: Schneidmüller, Bernd/Weinfurter, Stefan/Matheus, Michael et al. (Hg.): Die Päpste und die Einheit der lateinischen Welt. Bd. 1: Die Päpste. Amt und Herrschaft in Antike, Mittelalter und Renaissance. Mannheim: Schnell und Steiner, S. 357-383.

Kato, Hiloko (2015): Versehren, Verschandeln und Bekritzeln. Tabu(brüche) an den Rändern von Texten. In: Kodikas 38/3-4, S. 279-296.

Kaufmann, Thomas (2015): Reformation der Heiligenverehrung? In: Herbers, Klaus/Düchting, Larissa (Hg.): Sakralität und Devianz. Konstruktionen Normen - Praxis. Stuttgart: Franz Steiner, S. 209-230.

Klein, Peter K: Rand- oder Schwellenphänomen? Zur Deutung der Randbilder in der mittelalterlichen Kunst. In: Knefelkamp, Ulrich (Hg.): Grenze und Grenzüberschreitung im Mittelalter. 11. Symposium des Mediävistenverbandes vom 14. bis 17. März 2005 in Frankfurt an der Oder. Berlin: Akademie-Verlag, S. 166-187.

Koch, Elke (2017): Das Abenteuer im Paratext: Gabriel Rollenhagens Um-Rahmung der Brandan-Legende. In: Eming, Jutta/Schlechtweg-Jahn, Ralf (Hg.): Aventiure und Eskapade. Narrative des Abenteuerlichen vom Mittelalter zur Moderne. Göttingen: V \& R unipress, S. 49-62.

Kragl, Florian (2016): Die (Un-)Sichtbarkeit des Paratexts. Von einem Prinzip mittelalterlicher Buchgestaltung am Beispiel der ,Herzmaere'-Überlieferung. In: Beiträge zur Geschichte der deutschen Sprache und Literatur 138, S. 360-432.

Kuhn, Roman (2018): Wahre Geschichten, frei erfunden. Verhandlungen und Markierungen von Fiktion im Peritext. Berlin/Boston: De Gruyter.

Luckmann, Thomas (1986): Grundformen der gesellschaftlichen Vermittlung des Wissens: Kommunikative Gattungen. In: Kölner Zeitschrift für Soziologie und Sozialpsychologie. Sonderheft 27, S. 191-211.

Luhmann, Niklas (2000): Religion der Gesellschaft. Hrsg. von André Kieserling. Frankfurt am Main: Suhrkamp.

Luhmann, Niklas (2002): Die Kunst der Gesellschaft. Frankfurt am Main: Suhrkamp.

Luhmann, Niklas (2008): Das Medium der Kunst. In: Ders.: Schriften zu Kunst und Literatur. Hrsg. von Niels Werber. Frankfurt am Main: Suhrkamp, S. 123-138.

Menke, Bettine (2015): Text-Oberfläche. Figur und Grund, der Text und seine Ränder, Glossen, Kommentare. In: Lechtermann, Christina/Rieger, Stefan (Hg.): Das Wissen der Oberfläche. Epistemologie des Horizontalen und Strategien der Benachbarung. Zürich/Berlin: Diaphanes, S. 125-148.

Moulin, Claudine: Rand und Band. Über das Spurenlesen in Handschrift und Druck. In: Carmassi, Patrizia/ Heitzmann, Christian (Hg.): Marginalien in Bild und 
Text. Essays zu mittelalterlichen Handschriften. Wiesbaden: Harrassowitz Verlag, S. 19-59.

Münkler, Marina (2008): Sündhaftigkeit als Generator von Individualität. Zu den Transformationen legendarischen Erzählens in den Faustbüchern des 16. und 17. Jahrhunderts. In: Strohschneider, Peter $(\mathrm{Hg}$.$) : Literarische und religiöse Kommunikation in$ Mittelalter und Früher Neuzeit. DFG-Symposion 2006. Berlin/New York: De Gruyter, S. 25-61.

Münkler, Marina (2011): Narrative Ambiguität. Die Faustbücher des 16. bis 18. Jahrhunderts. Göttingen: Vandenhoeck \& Ruprecht 2011.

Münkler, Marina (2013): Amicus Dei. Konstruktionsformen des Heiligen am Beispiel der Franziskuslegenden. In: Vorländer, Hans (Hg.): Transzendenz und die Konstitution von Ordnungen. Berlin/New York: De Gruyter, S. 374-394.

Münkler, Marina (2015): Legende/Lügende. Die protestantische Polemik gegen die katholische Legende und Luthers Lügend von St. Johanne Chrysostomo. In: Schwerhoff, Gerd/Piltz, Eric (Hg.): Gottlosigkeit und Eigensinn. Religiöse Devianz im konfessionellen Zeitalter. Berlin: Duncker \& Humblot, S. 121-147.

Münkler (2019) Luthers Rom. Augenzeugenschaft, Invektivität und Konversion. In: Mauntel, Christoph/ Leppin, Volker (Hg.): Transformationen Roms in der Vormoderne. Basel/Stuttgart: Schwabe/W. Kohlhammer, S. 213-242.

Ott, Michael Ralf (2010): Die Erfindung des Paratextes. Überlegungen zur frühneuzeitlichen Textualität. URL: http://publikationen.ub.uni-frankfurt.de/frontdoor/ index/index/docId/7858 (letzter Zugriff: 21.11.2020).

Pfrunder, Peter (1989): Pfaffen, Ketzer, Totenfresser. Fastnachtskultur der Reformationszeit - Die Berner Spiele von Niklaus Manuel. Zürich: Chronos.

Sablotny, Antje (2019): Metalegende. Die protestantische Lügende als invektive Metagattung. In: Beiträge zur mediävistischen Erzählforschung 2, S. 148-200. DOI: https://doi.org/10.25619/BmE2019228 (letzter Zugriff 07.11.2021).

Sablotny, Antje (2020): Zeit und âventiure in Wolframs von Eschenbach Parzival. Zur narrativen Identitätskonstruktion des Helden. Berlin/Boston: De Gruyter.

Sablotny, Antje (2021): corpus corruptum: Die Herabsetzung heiliger Körper in den Papistischen Lügen Hieronymus Rauschers. In: Israel, Uwe/Müller, Jürgen (Hg.): Körper-Kränkungen. Der menschliche Leib als Medium der Herabsetzung. Frankfurt/New York: Campus.

Sablotny, Antje (im Erscheinen): Doing the Other. Zur kommunikativen Praxis der Grenzziehung in Erasmus Alberus' Barfuser Münche Eulenspiegel vnd Alcoran und Hieronymus Rauschers Papistischen Lügen. In: Blum, Daniela/Detering, Nicolas/Gunreben, Marie/Lüpke, Beatrice von (Hg.): Entscheidung zur Heiligkeit? Autonomie und Providenz im legendarischen Erzählen vom Mittelalter bis zur Gegenwart. Heidelberg: Winter.

Sánchez, Yvette (1999): Titel als Mittel. Poetologie eines Paratexts. In: arcadia 34/2, S. 244-261.
Schenda, Rudolf (1970): Die protestantisch-katholische Legendenpolemik im 16. Jahrhundert. In: Archiv für Kulturgeschichte 52, S. 28-48.

Schenda, Rudolf (1974): Hieronymus Rauscher und die protestantisch-katholische Legendenpolemik. In: Brückner, Wolfgang (Hg.): Volkserzählung und Reformation. Ein Handbuch zur Tradierung und Funktion von Erzählstoffen und Erzählliteratur im Protestantismus. Berlin: Erich Schmidt, S. 178-259.

Schlesier, Renate (1993): Art. Apotropäisch. In: Cancik, Hubert/Gladigow, Burkhard/Laubscher, Matthias (Hg.): Handbuch religionswissenschaftlicher Grundbegriffe. Bd. 2. Stuttgart/Berlin/Köln: W. Kohlhammer, S. 41-45.

Schmidt, Gabriela (2008): Pluralisierung von Autorschaft Entgrenzung des Textes. Die Fiktionalisierung des Paratextes in Thomas Mores literarischen Dialogen. In: Ammon, Frieder von/Vögel, Herfried (Hg.): Die Pluralisierung des Paratextes in der Frühen Neuzeit. Theorie, Formen, Funktionen. Berlin: LIT Verlag, S. 225-254.

Schnyder, André (1979): Legendenpolemik und Legendenkritik in der Reformation: Die Lügend von St. Johanne Chrysostomo bei Luther und Cochläus. Ein Beitrag zur Rezeption des Legendars Der Heiligen Leben. In: Archiv für Reformationsgeschichte 70, S. 122-140.

Schwitzgebel, Bärbel (1996): Noch nicht genug der Vorrede. Zur Vorrede volkssprachiger Sammlungen von Exempeln, Fabeln, Sprichwörtern und Schwänken des 16. Jahrhunderts. Tübingen: Max Niemeyer.

Stanitzek, Georg (2004): Texte, Paratexte, in Medien: Einleitung. In: Ders./Kreimeier, Klaus (Hg.): Paratexte in Literatur, Film, Fernsehen. Berlin: Akademie Verlag, S. 3-19.

Stenzel, Jürgen (1986): Rhetorischer Manichäismus. Vorschläge zu einer Theorie der Polemik. In: Koopmann, Helmut/Worstbrock, Franz Josef (Hg.): Formen und Formgeschichte des Streitens. Der Literaturstreit. Tübingen: Max Niemeyer 1986, S. 3-11.

Stollberg-Rilinger, Barbara (2013): Einleitung. In: Pietsch, Andreas/Dies. (Hg.): Konfessionelle Ambiguität. Uneindeutigkeit und Verstellung als religiöse Praxis in der Frühen Neuzeit. Heidelberg: Gütersloher Verlagshaus, S. 9-26.

Struth, Christiane (2014): Metagattungen als Paradigma literarischer Selbstreflexivität. In: Nünning, Ansgar/ Kovach, Elizabeth (Hg.): Key Concepts and New Topics in English and American Studies. Schlüsselkonzepte und neue Themen in der Anglistik und Amerikanistik. Trier: WVT, S. 263-281.

Struth, Christiane (2016): Die Metaautobiographie. Theorie, Poetik und Typologie eines neueren Genres der englischsprachigen Erzählliteratur. Trier: WVT.

Vollmann, Benedikt Konrad (2002): Erlaubte Fiktionalität: die Heiligenlegende. In: Knapp, Fritz Peter/Niesner, Manuela (Hg.): Historisches und fiktionales Erzählen im Mittelalter. Berlin: Duncker \& Humblot, S. 63-72.

Voßkamp, Wilhelm (1977): Gattungen als literarischsoziale Institutionen. In: Hinck, Walter (Hg.): 
Textsortenlehre - Gattungsgeschichte. Heidelberg: Quelle \& Meyer, S. 27-44.

Voßkamp, Wilhelm (1990): Utopie als Antwort auf Geschichte. Zur Typologie literarischer Utopien in der Neuzeit. In: Eggert, Hartmut/Profitlich, Ulrich/ Scherpe, Klaus R. (Hg.): Geschichte als Literatur. Formen und Grenzen der Repräsentation von Vergangenheit. Stuttgart: Metzler, S. 273-283.

Wagner, Bettina (2008): An der Wiege des Paratexts. Formen der Kommunikation zwischen Druckern, Herausgebern und Lesern im 15. Jahrhundert. In: Ammon, Frieder von/Vögel, Herfried (Hg.): Die Pluralisierung des Paratextes in der Frühen Neuzeit. Theorie, Formen, Funktionen. Berlin: LIT Verlag, S. 133-156.

Wirth, Uwe (2009): Paratext und Text als Übergangszone. In: Hallet, Wolfgang/ Neumann, Birgit (Hg.): Raum und Bewegung in der Literatur. Die Literaturwissenschaften und der Spatial Turn. Bielefeld: transcript, S. 167-177.

Wolf, Werner (1993): Ästhetische Illusion und Illusionsdurchbrechung in der Erzählkunst. Theorie und Geschichte mit Schwerpunkt auf englischem illusionsstörenden Erzählen. Tübingen: Max Niemeyer.

Wolf, Werner (2007): Metaisierung als transgenerisches und transmediales Phänomen. Ein Systematisierungsversuch metareferentieller Formen und Begriffe in Literatur und anderen Medien. In: Hauthal,
Janine/Nadj, Julijana/Nünning, Ansgar et al. (Hg.): Metaisierung in Literatur und anderen Medien. Theoretische Grundlagen, historische Perspektiven, Metagattungen, Funktionen. Berlin: De Gruyter, S. 25-64.

Wolf, Werner (2008): Prologe als Paratexte und/oder dramatische (Eingangs-)Rahmungen? ,Literarische Rahmung' als Alternative zum problematischen Paratext-Konzept. In: Ammon, Frieder von/Vögel, Herfried (Hg.): Die Pluralisierung des Paratextes in der Frühen Neuzeit. Theorie, Formen, Funktionen. Berlin: LIT Verlag, S. 79-98.

Ziegeler, Hans-Joachim (1999): Wahrheiten, Lügen, Fiktionen. Zu Martin Luthers, Lügend von S. Johanne Chrysostomo' und zum Status literarischer Gattungen im 15. und 16. Jahrhundert. In: Haug, Walter (Hg.): Mittelalter und frühe Neuzeit. Übergänge, Umbrüche und Neuansätze. Tübingen: Max Niemeyer, S. 237-262.

Zillien, Nicole (2019): Affordanz. In: Liggieri, Kevin/ Müller, Oliver (Hg.): Mensch-Maschine-Interaktion. Handbuch zu Geschichte - Kultur - Ethik. Berlin: Metzler/Springer Nature, Kap. III Begriffe und Konzepte, S. 226-228.

Zymner, Rüdiger (2003): Gattungstheorie. Probleme und Positionen der Literaturwissenschaft. Paderborn: mentis. 